\title{
Amphetamine Derivatives as Monoamine Oxidase Inhibitors
}

\author{
Miguel Reyes-Parada ${ }^{1,2^{*}}$, Patricio Iturriaga-Vasquez ${ }^{3}$ and Bruce K. Cassels ${ }^{4}$ \\ ${ }^{1}$ Centro de Investigación Biomédica y Aplicada (CIBAP), Escuela de Medicina, Facultad de Ciencias Médicas, Universidad \\ de Santiago de Chile, Santiago, Chile, ${ }^{2}$ Facultad de Ciencias de la Salud, Universidad Autónoma de Chile, Talca, Chile, \\ ${ }^{3}$ Departamento de Ciencias Químicas y Recursos Naturales, Facultad de Ingeniería y Ciencias, Universidad de la Frontera, \\ Temuco, Chile, ${ }^{4}$ Departamento de Química, Facultad de Ciencias, Universidad de Chile, Santiago, Chile
}

OPEN ACCESS

Edited by:

Juan J. Canales,

University of Tasmania, Australia

Reviewed by:

David Pubill,

University of Barcelona, Spain

Matthias E. Liechti,

University Hospital of Basel,

Switzerland

*Correspondence:

Miguel Reyes-Parada

miguel.reyes@usach.cl

Specialty section:

This article was submitted to

Neuropharmacology,

a section of the journal

Frontiers in Pharmacology

Received: 14 September 2019

Accepted: 09 December 2019

Published: 23 January 2020

Citation:

Reyes-Parada M, Iturriaga-Vasquez $P$ and Cassels BK (2020) Amphetamine

Derivatives as Monoamine

Oxidase Inhibitors.

Front. Pharmacol. 10:1590.

doi: 10.3389/fphar.2019.01590
Amphetamine and its derivatives exhibit a wide range of pharmacological activities, including psychostimulant, hallucinogenic, entactogenic, anorectic, or antidepressant effects. The mechanisms of action underlying these effects are usually related to the ability of the different amphetamines to interact with diverse monoamine transporters or receptors. Moreover, many of these compounds are also potent and selective monoamine oxidase inhibitors. In the present work, we review how structural modifications on the aromatic ring, the amino group and/or the aliphatic side chain of the parent scaffold, modulate the enzyme inhibitory properties of hundreds of amphetamine derivatives. Furthermore, we discuss how monoamine oxidase inhibition might influence the pharmacology of these compounds.

Keywords: monoamine oxidase, amphetamine derivatives, serotonin syndrome, serotonin transporter, dopamine transporter, norepinephrine transporter, monoamine oxidase-A

\section{INTRODUCTION}

Since its first description as "Phenisopropylamin" more than a century ago (Edeleano, 1887), amphetamine (1-phenylpropan-2-amine, phenylisopropylamine, amfetamine, alphamethylphenethylamine; AMPH; Figure 1) has received considerable attention due to its multiple psychotropic effects, first noted in the early 1930s (Prinzmetal and Bloomberg, 1935). Nowadays, AMPH is indicated for the treatment of attention deficit hyperactivity disorder (Heal et al., 2013), narcolepsy (Dauvilliers and Barateau, 2017), and - in the form of its prodrug lisdexamfetaminebinge-eating disorder (Hilbert, 2019). Its psychostimulant effects are usually related to its catecholamine-releasing properties, which arise from its ability to compete with dopamine (DA) and norepinephrine (NE) for uptake into the nerve terminals, and to induce reverse transport via the corresponding transporter (DAT and NET, respectively). Nevertheless, its polypharmacological

\footnotetext{
Abbreviations: AMPH, amphetamine; DA, dopamine; NE, norepinephrine; DAT, dopamine transporter; NET, norepinephrine transporter; 5-HT, serotonin; SERT, serotonin transporter; MAO, monoamine oxidase; FAD, flavin adenine dinucleotide; MAOI, monoamine oxidase inhibitor; MTA, 4-methylthioamphetamine; PMA, $p$ methoxyamphetamine; MDA, 3,4-methylenedioxyamphetamine; NMMTA, 4-methylthiomethamphetamine; PMMA, $p$ methoxymethamphetamine; MDMA, 3,4-methylenedioxymethamphetamine; FLA727, p-methylaminoamphetamine; MSOA, $p$-methylsulfoxyamphetamine; MSO2A, $p$-methylsulfonylamphetamine; PCA, $p$-chloroamphetamine; PBA, $p$ bromoamphetamine; PFA, p-fluoroamphetamine; FLA289, p-dimethylaminoamphetamine; NIPA, 2naphthylisopropylamine (also known as PAL-287); DOI, 4-iodo-2,5,-dimethoxyamphetamine; DOB, 4-bromo-2,5,dimethoxyamphetamine.
} 


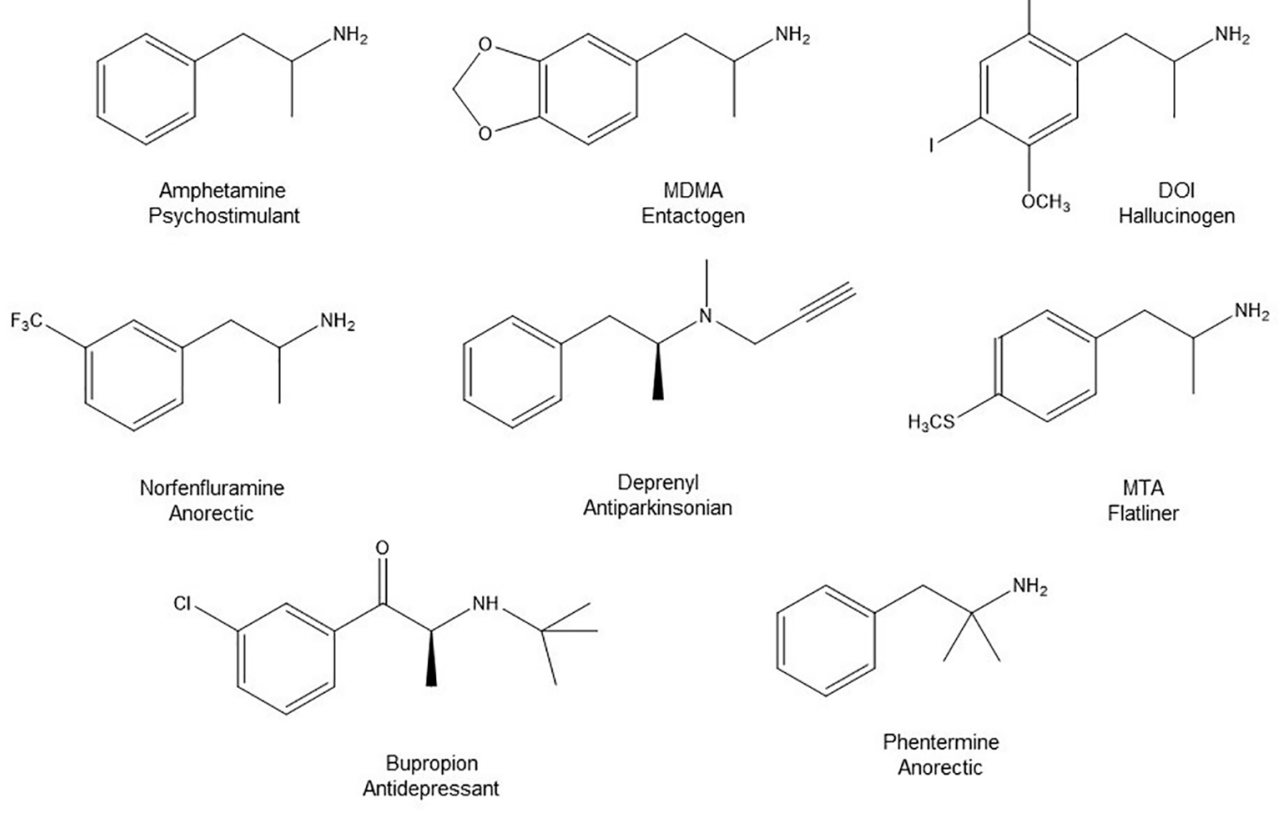

FIGURE 1 | Chemical structures of some AMPH derivatives.

profile involves actions upon other monoaminergic targets such as the serotonin (5-HT) transporter (SERT), the vesicular monoamine transporter and monoamine oxidase (MAO) (Sulzer et al., 2005; Heal et al., 2013).

A remarkable characteristic of AMPH is that subtle structural variations can produce drastic changes in its pharmacodynamics, and lead to compounds that interact differentially with several biogenic amine target proteins. Consequently, the AMPH skeleton has served as a privileged scaffold for the design and synthesis of hundreds of derivatives with many different and often useful activities, but also conveying misuse potential (Biel and Bopp, 1978; Nichols, 1994; Glennon, 1999; Rothman and Baumann, 2003; Welter-Luedeke and Maurer, 2016). Thus, the diversity of mechanisms of action of AMPH derivatives determines a many-colored palette of pharmacological activities in humans, including psychostimulant, entactogenic, psychedelic, anorectic, nootropic, and antidepressant effects. It is noteworthy that the structural changes also modify toxicological properties and abuse liability of AMPH derivatives (Fleckenstein et al., 2007; Rothman et al., 2007; Simmler et al., 2013; Barbosa et al., 2015).

MAO (monoamine oxygen oxidoreductase (deaminating) (flavin-containing); EC 1.4.3.4) is the main catabolic enzyme for biogenic monoamines such as NE, DA, 5-HT, and $\beta$ phenethylamine, and also for dietary and xenobiotic amines such as tyramine and benzylamine. MAO exists in two isoforms termed MAO-A and MAO-B. Both isozymes are outer mitochondrial membrane-bound flavoproteins, with the FAD cofactor covalently bound to the enzyme. The metabolic reaction involves the generation of an imine intermediate and the reduction of the flavin cofactor, which is reoxidized by molecular oxygen producing hydrogen peroxide. The imine intermediate is hydrolyzed, in a non-enzymatic process, generating ammonia and the corresponding aldehyde (Shih et al., 1999; Tipton et al., 2004; Edmondson et al., 2007). Although both isoforms have similar catalytic activities, they differ in their molecular genetics, physiological roles, tissue distribution, substrate preference, and inhibitor selectivity (Reyes-Parada et al., 2005). In the central nervous system, catecholaminergic neurons contain predominantly MAO-A, whereas serotonergic neurons express MAO-B (Westlund et al., 1988; Luque et al., 1995). MAO-A preferentially metabolizes $5-\mathrm{HT}$ and is irreversibly inhibited by nanomolar concentrations of clorgyline, whereas MAO-B preferentially catalyzes the oxidative deamination of phenethylamine and benzylamine and is irreversibly inhibited by nanomolar concentrations of $l$-deprenyl. DA and NE are nonselective substrates of both isoforms (Youdim et al., 2006). MAO inhibitors (MAOI) are currently used in the treatment of diverse neuropsychiatric and neurological disorders, including depression and Parkinson's disease (Cesura and Pletscher, 1992; Youdim et al., 2006; Finberg and Rabey, 2016; Kumar et al., 2017). In 2002, Binda and colleagues (Binda et al., 2002) published a seminal article showing the high-resolution structure of human MAO-B. Subsequent structures of this enzyme (Binda et al., 2003; Binda et al., 2004), as well as that of rat (Ma et al., 2004) and human MAO-A (De Colibus et al., 2005; Son et al., 2008), have allowed detailed comparison of the overall structures of both isoforms and their active sites (Binda et al., 2011; 
Iacovino et al., 2018). Thus, the substrate/inhibitor binding site of both isozymes (see Figure 2) can be described as a pocket lined by the isoalloxazine ring and several aliphatic and aromatic residues. A critical role of Y444, Y407, G215, and I180 of MAOA (Y435, Y398, G206, and L171 being the corresponding residues in MAO-B) in the orientation and stabilization of the substrate/ inhibitor binding can be inferred from the X-ray diffraction data. The availability of MAO crystal structures has allowed a quicker pace in the rational design of novel MAOIs and in the understanding of catalytic and inhibitory mechanisms. Thus, a vast number of studies in which molecular simulation approaches have been used to rationalize and/or to predict the functional interactions between the proteins and their substrates or inhibitors have been reported recently (Ferino et al., 2012; Vianello et al., 2016; Dhiman et al., 2017; Dhiman et al., 2018).

In the following pages, we review the effects of several dozen AMPH derivatives upon MAOs and describe, through the analysis of a set of representative examples, how structural

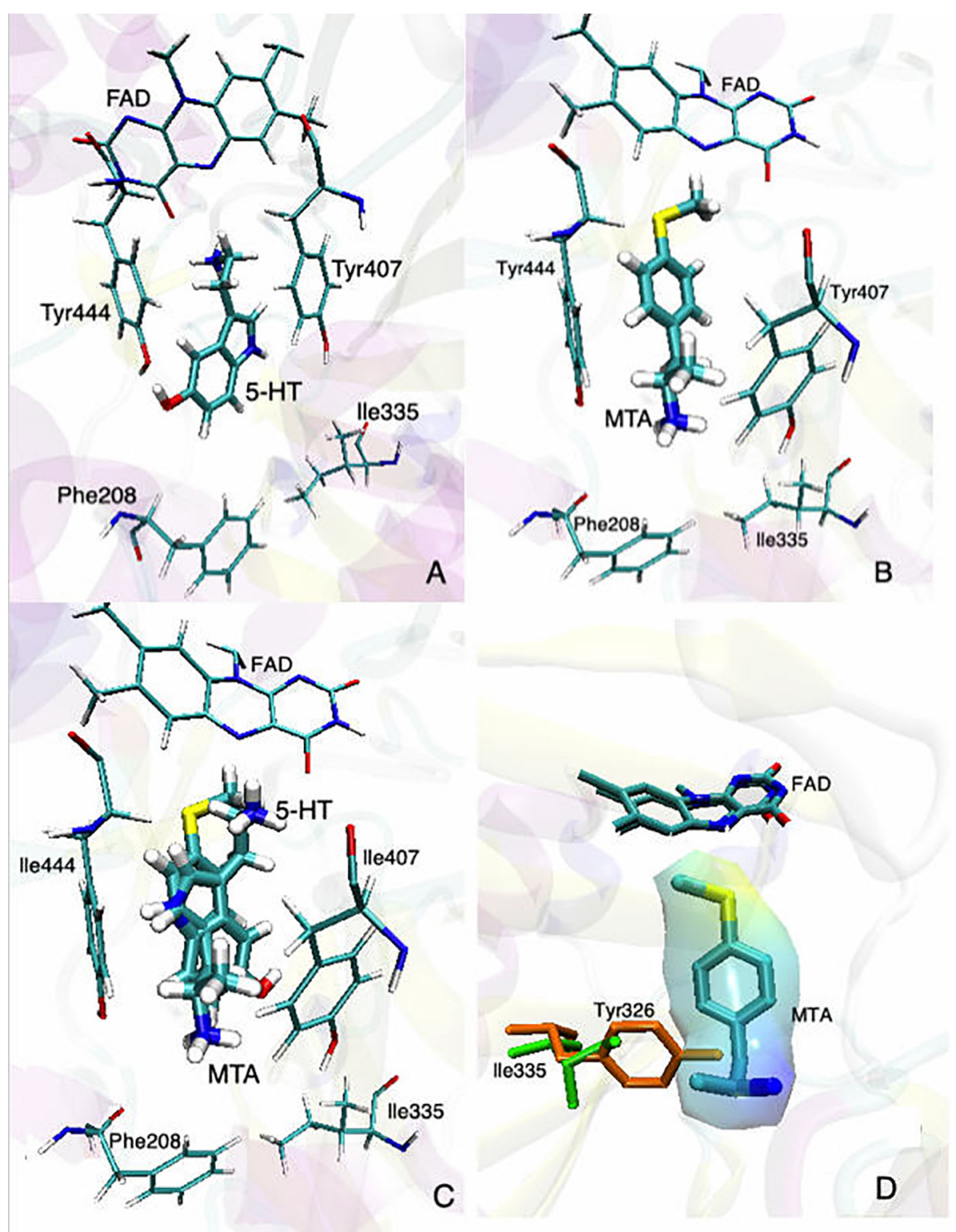

FIGURE 2 | Binding modes of (A) 5-HT and (B) MTA to MAO-A (PDB: 2BXS). (C) Superimposed structures of 5-HT and MTA docked into the active site of MAO-A (D) Superimposed binding sites of MAO-A (green residues) and MAO-B (orange residues) with MTA already docked into the active site of MAO-A; the "wrapper" around MTA represents the solvent accessible surface area (SASA). In all cases, for the sake of clarity, only the most relevant residues are shown. Docking conditions were as in Fierro et al., 2007. 
modifications on the aromatic ring, the amino group and/or the aliphatic side chain of the parent scaffold, modulate the enzyme inhibitory properties of this type of compounds.

\section{GENERAL NATURE OF MAO INHIBITION BY AMPH DERIVATIVES}

Since the first pharmacological study more than 50 years ago proving the existence of the two enzyme isoforms (Johnston, 1968), hundreds of AMPH derivatives have been tested as MAOIs. Tables 1-5 summarize the effects of a subset of these compounds. It should be noted that, as expected for results obtained from different laboratories over a long period of time, the methodological approaches used to assess MAO inhibition are diverse. Thus, a variety of biochemical assays to follow MAO activities (e.g. radiometric, luminometric, spectrophotometric, electrochemical, fluorometric), substrates (e.g. 5-HT, kynuramine, $\beta$ phenethylamine, benzylamine, 4-dimethylaminophenethylamine), inhibition parameters (e.g. $\mathrm{IC}_{50}, \mathrm{~K}_{\mathrm{i}}$ ), tissue sources (e.g. brain, lung, liver), and species (e.g. rat, mouse, human from fresh tissue, human recombinant heterologously expressed in yeast), have been employed in these experiments. Therefore, although we made an effort to consider results obtained under relatively similar conditions for comparative analysis, the reader should bear in mind this limitation when evaluating the data presented below.

Despite these considerations, some general conclusions about the MAO inhibitory activity of AMPH derivatives can be established. Thus, in the vast majority of the cases in which a relevant inhibitory activity was detected, this effect shows a clear selectivity towards MAO-A (e.g. Mantle et al., 1976; Green and el Hait, 1980; Ask et al., 1982a and Ask et al., 1982b; Ask et al., 1985; Florvall et al., 1986; Scorza et al., 1997; Gallardo-Godoy et al., 2005; Matsumoto et al., 2014; Tables 1-5). In addition, when assessed, AMPH derivatives produce in all cases a competitive and reversible ${ }^{1}$ inhibition of MAO (e.g. Mantle et al., 1976; Fowler and Oreland, 1981; Arai et al., 1990; Leonardi and Azmitia, 1994; Ulus et al., 2000; Fierro et al., 2007). Even though no crystal structure of MAO-A in complex with AMPH derivatives has been reported yet, docking simulations have shed light on the molecular mechanism underlying both the MAO-A inhibitory activity and the selectivity exhibited by these compounds (Vallejos et al., 2005; Fierro et al., 2007; Vilches-Herrera et al., 2009 and VilchesHerrera et al., 2016). Figure 2 summarizes our current view in

\footnotetext{
${ }^{1}$ It should be noted that we do not review here irreversible inhibitors such as deprenyl or tranylcypromine. These compounds and their analogues can also be considered as AMPH derivatives, but due to particular structural characteristics (the presence either of a propargyl moiety on the amino group or a cyclopropyl ring involving the $\alpha$ and $\beta$ carbons of the side chain, respectively), they are suicide substrates that can form covalent adducts with the flavin ring of the enzyme's cofactor. This characteristic leads to an irreversible mode of inhibition and generates completely different structure-activity relationships. These have been analyzed elsewhere (e.g. Magyar, 1994; Yoshida et al., 2004; Malcomson et al., 2015) and the reader is also referred to the literature (Binda et al., 2003; De Colibus et al., 2005) to have insights regarding the actual structural characteristics of the MAOdrug adducts formed by these compounds.
}

this regard. Thus, when a substrate, in this case $5-\mathrm{HT}$, is docked at the catalytic site, it locates in a pose where the amino group is in close proximity to the isoalloxazine ring of the FAD cofactor (Figure 2A). This would favor the abstraction of the pro- $R \alpha$ proton of the amine by the N5 atom of the flavin ring, which is a critical step of MAO-catalyzed amine oxidation. On the other hand, AMPH derivatives (exemplified in this case by 4methylthioAMPH; MTA) docked at the same site, exhibit binding modes where the amino group points away from the FAD ring system (Figure 2B) but with the aromatic ring positioned almost identically to that of the substrate (Figure 2C). Indeed, such a binding mode provides a rationale for the observed inhibitory activity, since while blocking the access of any substrate to the active site, AMPH derivatives could avoid deamination by adopting a pose where the amino group is remote from the influence of the flavin ring. Furthermore, in Figure 2D the active site of MAO-B was superimposed on the corresponding site of MAO-A already docked with MTA. As shown, the presence of Y326 in MAO-B (I335 being the corresponding residue in $\mathrm{MAO}-\mathrm{A}$ ), could prevent the close fit of MTA into the active site of MAO-B. Thus, our docking experiments suggest a possible explanation for the MAO-A selectivity exhibited by most AMPH derivatives. It is worth pointing out that fragments $\mathrm{I} 335$ and Y326 in MAO-A and MAO-B, respectively, have been regarded as major determinants of selectivity for both substrates and inhibitors (Edmondson et al., 2007; Binda et al., 2011; Iacovino et al., 2018).

\section{STRUCTURE-ACTIVITY RELATIONSHIPS OF AMPH DERIVATIVES AS MAOI}

\section{Modifications of the Side Chain}

The presence of a methyl group on the $\alpha$-carbon atom of phenethylamine transforms this compound, which is a selective MAO-B substrate, into AMPH which is a selective MAO-A inhibitor. This substrate-to-inhibitor change has also been reported for other phenethylamine/AMPH derivative pairs (e.g. Edwards, 1978; Yu, 1986; Fagervall et al., 1988; ReyesParada et al., 1994a and Reyes-Parada et al., 1994b; Tables 12. Figure 3). Considering that the $\alpha-\mathrm{C}-\mathrm{H}$ bond cleavage is likely the rate-limiting step in the catalytic cycle of both MAO isozymes (Miller and Edmondson, 1999), it seems reasonable that impeding/altering the feasibility of this step results in MAO inhibitory properties. Although stereospecific abstraction of the pro- $R$-hydrogen has been demonstrated with several substrates (Yu et al., 1986; Yu, 1988), it is noteworthy that both isomers of chiral AMPH derivatives inhibit MAOs, regardless of whether the pro- $R$ - or the pro-S-hydrogen is the substituted atom (see Tables 1-4 and references therein). This supports our idea that MAOI properties of AMPH derivatives are not necessarily due to the hindrance of hydrogen abstraction, but that the introduction of alkyl substituents at the $\alpha$-carbon atom of the phenethylamine results in its adopting a different binding mode in the catalytic site (Figure 2). Nevertheless, extension of the alkyl substituent on the $\alpha$-carbon atom or the introduction of a second methyl 


\begin{tabular}{|c|c|c|c|c|c|c|c|c|c|c|c|}
\hline \multirow[b]{2}{*}{ Compound $^{\mathrm{b}}$} & \multirow[b]{2}{*}{$\mathbf{R 2}$} & \multirow[b]{2}{*}{ R3 } & \multirow[b]{2}{*}{ R4 } & \multirow[b]{2}{*}{ R5 } & \multirow[b]{2}{*}{ R6 } & \multirow[b]{2}{*}{$R^{\alpha 1}$} & \multirow[b]{2}{*}{$\mathbf{R}^{\alpha 2}$} & \multirow[t]{2}{*}{$\begin{array}{l}\mathrm{RN2} \\
\mathbf{R}^{\mathrm{N} 1}\end{array}$} & \multirow[b]{2}{*}{$\mathbf{R}^{\mathrm{N} 2}$} & \multicolumn{2}{|c|}{$\begin{array}{l}\text { MAOI Activity } \\
\text { IC }_{50}(\mathrm{Ki})^{\mathrm{a}}(\mu \mathrm{M})\end{array}$} \\
\hline & & & & & & & & & & MAO-A & MAO-B \\
\hline (+)-Amphetamine & $\mathrm{H}$ & $\mathrm{H}$ & $\mathrm{H}$ & $\mathrm{H}$ & $\mathrm{H}$ & $\mathrm{CH}_{3}$ & $\mathrm{H}$ & $\mathrm{H}$ & $\mathrm{H}$ & $20.0^{c} ; 4.9^{d} ; 33.8^{e}$ & $770^{\mathrm{c}} ; 118^{\mathrm{d}} ; 161^{\mathrm{e}}$ \\
\hline Amphetamine & $\mathrm{H}$ & $\mathrm{H}$ & $\mathrm{H}$ & $\mathrm{H}$ & $\mathrm{H}$ & \multicolumn{2}{|c|}{$\mathrm{HCH}_{3}$} & $\mathrm{H}$ & $\mathrm{H}$ & $11.0^{f}\left(5.3^{g}\right)$ & $236^{9}$ \\
\hline (-)-Amphetamine & $\mathrm{H}$ & $\mathrm{H}$ & $\mathrm{H}$ & $\mathrm{H}$ & $\mathrm{H}$ & $\mathrm{H}$ & $\mathrm{CH}_{3}$ & $\mathrm{H}$ & $\mathrm{H}$ & $70.0^{\mathrm{c}} ; 203^{\mathrm{e}}$ & $600^{\mathrm{c}} ; 180^{\mathrm{e}}$ \\
\hline Methamphetamine & $\mathrm{H}$ & $\mathrm{H}$ & $\mathrm{H}$ & $\mathrm{H}$ & $\mathrm{H}$ & \multicolumn{2}{|c|}{$\mathrm{HCH}_{3}$} & $\mathrm{CH}_{3}$ & $\mathrm{H}$ & $41^{h}\left(17.2^{g}\right)$ & $>200^{\mathrm{h}}(297)^{\mathrm{g}}$ \\
\hline Phentermine & $\mathrm{H}$ & $\mathrm{H}$ & $\mathrm{H}$ & $\mathrm{H}$ & $\mathrm{H}$ & $\mathrm{CH}_{3}$ & $\mathrm{CH}_{3}$ & $\mathrm{H}$ & $\mathrm{H}$ & $143^{i}\left(88^{d} ; 196^{g}\right)$ & $285^{\mathrm{i}}\left(310^{\mathrm{d}} ; 138^{\mathrm{g}}\right)$ \\
\hline AEPEA & $\mathrm{H}$ & $\mathrm{H}$ & $\mathrm{H}$ & $\mathrm{H}$ & $\mathrm{H}$ & \multicolumn{2}{|c|}{$\mathrm{HCH}_{2} \mathrm{CH}_{3}$} & $\mathrm{H}$ & $\mathrm{H}$ & $14.0^{9}$ & $234^{9}$ \\
\hline$N, \alpha$-DEPEA & $\mathrm{H}$ & $\mathrm{H}$ & $\mathrm{H}$ & $\mathrm{H}$ & $\mathrm{H}$ & \multicolumn{2}{|c|}{$\mathrm{HCH}_{2} \mathrm{CH}_{3}$} & $\mathrm{CH}_{2} \mathrm{CH}_{3}$ & $\mathrm{H}$ & $251^{9}$ & $159^{9}$ \\
\hline Amiflamine/(+)-FLA336 & $\mathrm{CH}_{3}$ & $\mathrm{H}$ & $\mathrm{N}\left(\mathrm{CH}_{3}\right)_{2}$ & $\mathrm{H}$ & $\mathrm{H}$ & $\mathrm{CH}_{3}$ & $\mathrm{H}$ & $\mathrm{H}$ & $\mathrm{H}$ & $0.8^{j} ; 2.0^{f}$ & $>1000^{j}$ \\
\hline FLA336 & $\mathrm{CH}_{3}$ & $\mathrm{H}$ & $\mathrm{N}\left(\mathrm{CH}_{3}\right)_{2}$ & $\mathrm{H}$ & $\mathrm{H}$ & \multicolumn{2}{|c|}{$\mathrm{HCH}_{3}$} & $\mathrm{H}$ & $\mathrm{H}$ & $2.7^{\mathrm{k}}$ & $440^{k}$ \\
\hline$(-)-$ FLA336 & $\mathrm{CH}_{3}$ & $\mathrm{H}$ & $\mathrm{N}\left(\mathrm{CH}_{3}\right)_{2}$ & $\mathrm{H}$ & $\mathrm{H}$ & $\mathrm{H}$ & $\mathrm{CH}_{3}$ & $\mathrm{H}$ & $\mathrm{H}$ & $3.0^{j}$ & $125^{\prime}$ \\
\hline FLA289 & $\mathrm{H}$ & $\mathrm{H}$ & $\mathrm{N}\left(\mathrm{CH}_{3}\right)_{2}$ & $\mathrm{H}$ & $\mathrm{H}$ & \multicolumn{2}{|c|}{$\mathrm{HCH}_{3}$} & $\mathrm{H}$ & $\mathrm{H}$ & $3.7^{\mathrm{l}} ; 2.0^{\mathrm{m}}$ & $400^{\prime}$ \\
\hline FLA727 & $\mathrm{H}$ & $\mathrm{H}$ & $\mathrm{NHCH}_{3}$ & $\mathrm{H}$ & $\mathrm{H}$ & \multicolumn{2}{|c|}{$\mathrm{HCH}_{3}$} & $\mathrm{H}$ & $\mathrm{H}$ & $0.55^{1}-1.2^{m}$ & $1500^{\prime}$ \\
\hline (+)-FLA788 & $\mathrm{CH}_{3}$ & $\mathrm{H}$ & $\mathrm{NHCH}_{3}$ & $\mathrm{H}$ & $\mathrm{H}$ & $\mathrm{CH}_{3}$ & $\mathrm{H}$ & $\mathrm{H}$ & $\mathrm{H}$ & $0.13^{j}$ & $>1000^{j}$ \\
\hline FLA558 & F & $\mathrm{H}$ & $\mathrm{N}\left(\mathrm{CH}_{3}\right)_{2}$ & $\mathrm{H}$ & $\mathrm{H}$ & \multicolumn{2}{|c|}{$\mathrm{HCH}_{3}$} & $\mathrm{H}$ & $\mathrm{H}$ & $1.2^{\mathrm{k}}$ & $120^{k}$ \\
\hline FLA314 & $\mathrm{Cl}$ & $\mathrm{H}$ & $\mathrm{N}\left(\mathrm{CH}_{3}\right)_{2}$ & $\mathrm{H}$ & $\mathrm{H}$ & \multicolumn{2}{|c|}{$\mathrm{HCH}_{3}$} & $\mathrm{H}$ & $\mathrm{H}$ & $0.21^{k}$ & $80^{\mathrm{k}}$ \\
\hline FLA405 & $\mathrm{Br}$ & $\mathrm{H}$ & $\mathrm{N}\left(\mathrm{CH}_{3}\right)_{2}$ & $\mathrm{H}$ & $\mathrm{H}$ & \multicolumn{2}{|c|}{$\mathrm{HCH}_{3}$} & $\mathrm{H}$ & $\mathrm{H}$ & $0.22^{k}$ & $100^{k}$ \\
\hline FLA365 & $\mathrm{Cl}$ & $\mathrm{H}$ & $\mathrm{N}\left(\mathrm{CH}_{3}\right)_{2}$ & $\mathrm{H}$ & $\mathrm{Cl}$ & \multicolumn{2}{|c|}{$\mathrm{HCH}_{3}$} & $\mathrm{H}$ & $\mathrm{H}$ & $0.013^{1}$ & $180^{\prime}$ \\
\hline FLA450 & $\mathrm{Cl}$ & $\mathrm{H}$ & $\mathrm{N}\left(\mathrm{CH}_{3}\right)_{2}$ & $\mathrm{H}$ & $\mathrm{H}$ & \multicolumn{2}{|c|}{$\mathrm{HCH}_{2} \mathrm{CH}_{3}$} & $\mathrm{H}$ & $\mathrm{H}$ & $0.38^{k}$ & $75^{\mathrm{k}}$ \\
\hline FLA463 & $\mathrm{Cl}$ & $\mathrm{H}$ & $\mathrm{N}\left(\mathrm{CH}_{3}\right)_{2}$ & $\mathrm{H}$ & $\mathrm{H}$ & $\mathrm{CH}_{3}$ & $\mathrm{CH}_{3}$ & $\mathrm{H}$ & $\mathrm{H}$ & $1.2^{\mathrm{k}}$ & $700^{k}$ \\
\hline FLA717 & $\mathrm{CH}_{3}$ & $\mathrm{H}$ & $\mathrm{N}\left(\mathrm{CH}_{3}\right)_{2}$ & $\mathrm{H}$ & $\mathrm{H}$ & $\mathrm{CH}_{3}$ & $\mathrm{CH}_{3}$ & $\mathrm{H}$ & $\mathrm{H}$ & $12.0^{k}$ & $2100^{k}$ \\
\hline FLA384 & $\mathrm{H}$ & $\mathrm{CH}_{3}$ & $\mathrm{~N}\left(\mathrm{CH}_{3}\right)_{2}$ & $\mathrm{H}$ & $\mathrm{H}$ & \multicolumn{2}{|c|}{$\mathrm{HCH}_{3}$} & $\mathrm{H}$ & $\mathrm{H}$ & $8.0^{1}$ & $650^{\prime}$ \\
\hline (+)NBFOO3 & $\mathrm{CH}_{3}$ & $\mathrm{H}$ & $\mathrm{N}\left(\mathrm{CH}_{3}\right)_{2}$ & $\mathrm{Br}$ & $\mathrm{H}$ & $\mathrm{CH}_{3}$ & $\mathrm{H}$ & $\mathrm{H}$ & $\mathrm{H}$ & $1.1^{1}$ & $480^{\prime}$ \\
\hline
\end{tabular}

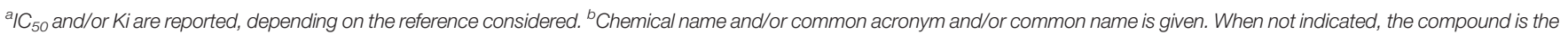
racemic mixture. ${ }^{C}$ Mantle et al., 1976. ${ }^{d}$ Ulus et al., 2000. ${ }^{\circledR}$ Robinson, $1985 .{ }^{f}$ Scorza et al., 1997. ${ }^{9}$ Santillo, 2014. ${ }^{h}$ Matsumoto et al., $2014 .{ }^{i}$ Kilpatrick et al., 2001. ${ }^{j}$ Ask et al., 1982b. ${ }^{k}$ Ask et al., 1982a. 'Ask et al., 1985. ${ }^{m}$ Reyes-Parada et al., 1994a.

group at this position, lead to a decrease of MAOI-A potency compared with the parent compounds (Figure 4). Moreover, cyclization of the side chain to generate 2 -aminoindan or 2aminotetraline analogues results in a marked decrease of the affinity of these compounds for MAOs (Feenstra et al., 1983; Scorza et al., 1999). Such reductions of inhibitory activity would presumably reflect impediments to binding in the enzyme's active site.

As shown in Tables 1, 2, and 4, in most of the instances in which the dependence of enzyme inhibition on the chirality of the $\alpha$-carbon atom has been tested, it has been found that the $(S)$ (+)-AMPH derivatives are the eutomers as MAOI-A (Mantle et al., 1976; Fowler and Oreland, 1981; Ask et al., 1982b; Robinson, 1985; Leonardi and Azmitia, 1994; HurtadoGuzmán et al., 2003; Figure 5). As stated before, the difference in MAO-A inhibitory potency between optical isomers of AMPH derivatives is, in general, not remarkable (Tables 1-4). This suggests that stereoselectivity may not be as influential in the pharmacodynamics of these compounds as has been shown to be for the effects of AMPH derivatives upon other monoaminergic target proteins such as monoamine transporters or 5-HT receptors (Nichols, 1994; Nichols, 2018).

Given the notoriety that the recreational use of cathinone derivatives has reached in the last few years (Simmler et al., 2013; Paillet-Loilier et al., 2014; Angoa-Pérez et al., 2017), it is somewhat surprising that MAOI properties of this type of compounds (as an example of $\beta$-substituted AMPH derivatives) have not been extensively studied. Nevertheless, Osorio-Olivares et al. (2004) reported that cathinone is almost completely devoid of activity as a MAOI, whereas some derivatives with alkylthio or alkoxy groups at the para position of the aromatic ring have $\mathrm{IC}_{50}$ values in the low micromolar range (Table 3). These results indicate that $\beta$-keto substitution of AMPH may lead to a decrease in MAOI-A potency (Figure 6). Nevertheless, some of these compounds showed an interesting MAO-B inhibiting activity (Table 3 ), which suggests that selectivity can be effectively modulated by side-chain substituents. In addition, $\beta$-keto substitution seems to diminish the enantioselectivity for MAO inhibition, since in some cases in which it was evaluated, the $R(-)$-derivatives were the eutomers 
TABLE 2 | MAO inhibitory activity of AMPH derivatives monosubstituted in the aromatic ring.

\begin{tabular}{|c|c|c|c|c|c|c|c|c|c|c|c|}
\hline \multirow[b]{2}{*}{ Compound $^{b}$} & \multirow[b]{2}{*}{ R2 } & \multirow[b]{2}{*}{ R3 } & \multirow[t]{2}{*}{ C- } & \multirow[t]{2}{*}{${ }_{\mathrm{R} 5}^{\mathrm{R} 2}$} & \multirow[b]{2}{*}{ R6 } & $\mathrm{R} \alpha 2$ & \multirow[t]{2}{*}{$\begin{array}{l}\mathrm{R} \alpha 1 \\
\mathrm{R}^{\alpha 2}\end{array}$} & \multirow[t]{2}{*}{$\begin{array}{l}\text { RN2 } \\
\\
\mathbf{R}^{\mathrm{N} 1}\end{array}$} & \multirow[b]{2}{*}{$\mathrm{R}^{\mathrm{N} 2}$} & $\begin{array}{l}\text { MAOI A } \\
I_{50}(\mathrm{Ki})\end{array}$ & $\begin{array}{l}\text { vity } \\
\text { M) }\end{array}$ \\
\hline & & & & & & $R^{\alpha 1}$ & & & & MAO-A & MAO-B \\
\hline PMA/4-MeOA & $\mathrm{H}$ & $\mathrm{H}$ & $\mathrm{OCH}_{3}$ & $\mathrm{H}$ & $\mathrm{H}$ & \multicolumn{2}{|c|}{$\mathrm{HCH}_{3}$} & $\mathrm{H}$ & $\mathrm{H}$ & $0.3^{\mathrm{c}} ; 0.6^{\mathrm{d}}\left(0.2^{\mathrm{e}}\right)$ & $45^{\mathrm{d}}\left(530^{\mathrm{e}}\right)$ \\
\hline 2-MeOA & $\mathrm{OCH}_{3}$ & $\mathrm{H}$ & $\mathrm{H}$ & $\mathrm{H}$ & $\mathrm{H}$ & \multicolumn{2}{|c|}{$\mathrm{HCH}_{3}$} & $\mathrm{H}$ & $\mathrm{H}$ & $9.0^{\mathrm{e}}$ & $350^{\mathrm{e}}$ \\
\hline 3-MeOA & $\mathrm{H}$ & $\mathrm{OCH}_{3}$ & $\mathrm{H}$ & $\mathrm{H}$ & $\mathrm{H}$ & \multicolumn{2}{|c|}{$\mathrm{HCH}_{3}$} & $\mathrm{H}$ & $\mathrm{H}$ & $23^{e}$ & $1940^{e}$ \\
\hline PMMA & $\mathrm{H}$ & $\mathrm{H}$ & $\mathrm{OCH}_{3}$ & $\mathrm{H}$ & $\mathrm{H}$ & \multicolumn{2}{|c|}{$\mathrm{HCH}_{3}$} & $\mathrm{CH}_{3}$ & $\mathrm{H}$ & $1.7^{d}$ & $58^{d}$ \\
\hline 4-EtOA & $\mathrm{H}$ & $\mathrm{H}$ & $\mathrm{OCH}_{2} \mathrm{CH}_{3}$ & $\mathrm{H}$ & $\mathrm{H}$ & \multicolumn{2}{|c|}{$\mathrm{HCH}_{3}$} & $\mathrm{H}$ & $\mathrm{H}$ & $0.22^{f}$ & $>100^{f}$ \\
\hline 4-PrOA & $\mathrm{H}$ & $\mathrm{H}$ & $\mathrm{O}\left(\mathrm{CH}_{2}\right)_{2} \mathrm{CH}_{3}$ & $H$ & $H$ & \multicolumn{2}{|c|}{$\mathrm{HCH}_{3}$} & $\mathrm{H}$ & $\mathrm{H}$ & $0.13^{f}$ & $>100^{f}$ \\
\hline 4-BuOA & $\mathrm{H}$ & $\mathrm{H}$ & $\mathrm{O}\left(\mathrm{CH}_{2}\right)_{3} \mathrm{CH}_{3}$ & $\mathrm{H}$ & $H$ & \multicolumn{2}{|c|}{$\mathrm{HCH}_{3}$} & $\mathrm{H}$ & $\mathrm{H}$ & $0.32^{f}$ & $>100^{f}$ \\
\hline 4-BzOA & $\mathrm{H}$ & $\mathrm{H}$ & $\mathrm{OCH}_{2}$ Phe & $\mathrm{H}$ & $H$ & \multicolumn{2}{|c|}{$\mathrm{HCH}_{3}$} & $\mathrm{H}$ & $\mathrm{H}$ & $3.42^{f}$ & $0.71^{f}$ \\
\hline MTA & $\mathrm{H}$ & $\mathrm{H}$ & $\mathrm{SCH}_{3}$ & $\mathrm{H}$ & $\mathrm{H}$ & \multicolumn{2}{|c|}{$\mathrm{HCH}_{3}$} & $\mathrm{H}$ & $\mathrm{H}$ & $0.25^{\mathrm{g}}$ & $\mathrm{NE}^{\mathrm{g}}$ \\
\hline$(+)-M T A$ & $\mathrm{H}$ & $\mathrm{H}$ & $\mathrm{SCH}_{3}$ & $\mathrm{H}$ & $\mathrm{H}$ & $\mathrm{CH}_{3}$ & $\mathrm{H}$ & $\mathrm{H}$ & $\mathrm{H}$ & $0.13^{h}$ & $N E^{h}$ \\
\hline$(-)-M T A$ & $\mathrm{H}$ & $\mathrm{H}$ & $\mathrm{SCH}_{3}$ & $H$ & $\mathrm{H}$ & $\mathrm{H}$ & $\mathrm{CH}_{3}$ & $\mathrm{H}$ & $\mathrm{H}$ & $2.04^{\mathrm{g}}$ & $N E^{g}$ \\
\hline NMMTA & $\mathrm{H}$ & $\mathrm{H}$ & $\mathrm{SCH}_{3}$ & $\mathrm{H}$ & $\mathrm{H}$ & \multicolumn{2}{|c|}{$\mathrm{HCH}_{3}$} & $\mathrm{CH}_{3}$ & $\mathrm{H}$ & $0.89^{9}$ & $N E^{g}$ \\
\hline DMMTA & $\mathrm{H}$ & $\mathrm{H}$ & $\mathrm{SCH}_{3}$ & $\mathrm{H}$ & $\mathrm{H}$ & \multicolumn{2}{|c|}{$\mathrm{HCH}_{3}$} & $\mathrm{CH}_{3}$ & $\mathrm{CH}_{3}$ & $2.10^{\mathrm{g}}$ & $\mathrm{NE}^{\mathrm{g}}$ \\
\hline NEMTA & $\mathrm{H}$ & $\mathrm{H}$ & $\mathrm{SCH}_{3}$ & $\mathrm{H}$ & $\mathrm{H}$ & \multicolumn{2}{|c|}{$\mathrm{HCH}_{3}$} & $\mathrm{CH}_{2} \mathrm{CH}_{3}$ & $\mathrm{H}$ & $1.80^{\mathrm{g}}$ & $\mathrm{NE}^{\mathrm{g}}$ \\
\hline DEMTA & $\mathrm{H}$ & $\mathrm{H}$ & $\mathrm{SCH}_{3}$ & $\mathrm{H}$ & $\mathrm{H}$ & \multicolumn{2}{|c|}{$\mathrm{HCH}_{3}$} & $\mathrm{CH}_{2} \mathrm{CH}_{3}$ & $\mathrm{CH}_{2} \mathrm{CH}_{3}$ & $6.45^{\mathrm{g}}$ & $\mathrm{NE}^{\mathrm{g}}$ \\
\hline NPMTA & $\mathrm{H}$ & $\mathrm{H}$ & $\mathrm{SCH}_{3}$ & $\mathrm{H}$ & $\mathrm{H}$ & \multicolumn{2}{|c|}{$\mathrm{HCH}_{3}$} & $\left(\mathrm{CH}_{2}\right)_{2} \mathrm{CH}_{3}$ & $\mathrm{H}$ & $2.41^{\mathrm{g}}$ & $>10^{g}$ \\
\hline DPMTA & $\mathrm{H}$ & $\mathrm{H}$ & $\mathrm{SCH}_{3}$ & $\mathrm{H}$ & $\mathrm{H}$ & \multicolumn{2}{|c|}{$\mathrm{HCH}_{3}$} & $\left(\mathrm{CH}_{2}\right)_{2} \mathrm{CH}_{3}$ & $\left(\mathrm{CH}_{2}\right)_{2} \mathrm{CH}_{3}$ & $>10^{9}$ & $\mathrm{NE}^{\mathrm{g}}$ \\
\hline NBzMTA & $\mathrm{H}$ & $\mathrm{H}$ & $\mathrm{SCH}_{3}$ & $\mathrm{H}$ & $\mathrm{H}$ & & & $\mathrm{CH}_{2}$ Phe & $\mathrm{H}$ & $>100^{f}$ & $>100^{f}$ \\
\hline MTAB & $\mathrm{H}$ & $\mathrm{H}$ & $\mathrm{SCH}_{3}$ & $H$ & $\mathrm{H}$ & $\mathrm{HCH}$ & $\mathrm{CH}_{3}$ & $\mathrm{H}$ & $\mathrm{H}$ & $0.84^{\mathrm{g}}$ & $\mathrm{NE}^{\mathrm{g}}$ \\
\hline ETA & $\mathrm{H}$ & $\mathrm{H}$ & $\mathrm{SCH}_{2} \mathrm{CH}_{3}$ & $\mathrm{H}$ & $\mathrm{H}$ & $\mathrm{HCH}$ & $\mathrm{CH}_{3}$ & $\mathrm{H}$ & $\mathrm{H}$ & $0.10^{\mathrm{C}}$ & $29^{c}$ \\
\hline$(+)$-ETA & $\mathrm{H}$ & $\mathrm{H}$ & $\mathrm{SCH}_{2} \mathrm{CH}_{3}$ & $\mathrm{H}$ & $\mathrm{H}$ & $\mathrm{CH}_{3}$ & $\mathrm{H}$ & $\mathrm{H}$ & $\mathrm{H}$ & $0.075^{\mathrm{h}}$ & $>100^{h}$ \\
\hline (+)-PTA & $\mathrm{H}$ & $\mathrm{H}$ & $\mathrm{S}\left(\mathrm{CH}_{2}\right)_{2} \mathrm{CH}_{3}$ & $\mathrm{H}$ & $\mathrm{H}$ & $\mathrm{CH}_{3}$ & $\mathrm{H}$ & $\mathrm{H}$ & $\mathrm{H}$ & $0.030^{h}$ & $14.0^{h}$ \\
\hline ITA & $\mathrm{H}$ & $\mathrm{H}$ & $\mathrm{SCH}\left(\mathrm{CH}_{3}\right)_{2}$ & $\mathrm{H}$ & $\mathrm{H}$ & & & $\mathrm{H}$ & $\mathrm{H}$ & $0.40^{\mathrm{C}}$ & $8.1^{c}$ \\
\hline (+)-BTA & $\mathrm{H}$ & $\mathrm{H}$ & $\mathrm{S}\left(\mathrm{CH}_{2}\right)_{3} \mathrm{CH}_{3}$ & $\mathrm{H}$ & $\mathrm{H}$ & & & $\mathrm{H}$ & $\mathrm{H}$ & $0.022^{h}$ & $4.6^{h}$ \\
\hline MSOA & $\mathrm{H}$ & $\mathrm{H}$ & $\mathrm{SOCH}_{3}$ & $\mathrm{H}$ & $\mathrm{H}$ & & & $\mathrm{H}$ & $\mathrm{H}$ & $>100^{i}$ & NT \\
\hline $\mathrm{MSO} 2 \mathrm{~A}$ & $\mathrm{H}$ & $\mathrm{H}$ & $\mathrm{SO}_{2} \mathrm{CH}_{3}$ & $\mathrm{H}$ & $\mathrm{H}$ & & & $\mathrm{H}$ & $\mathrm{H}$ & $>100^{i}$ & NT \\
\hline PCA/p-Chloroamphetamine & $\mathrm{H}$ & $\mathrm{H}$ & $\mathrm{Cl}$ & $H$ & $\mathrm{H}$ & & & $\mathrm{H}$ & $\mathrm{H}$ & $4.0^{c} ; 1.9^{j}$ & $\mathrm{NE}^{\mathrm{C}}$ \\
\hline PBA/p-Bromoamphetamine & $\mathrm{H}$ & $\mathrm{H}$ & $\mathrm{Br}$ & $\mathrm{H}$ & $\mathrm{H}$ & & & $\mathrm{H}$ & $\mathrm{H}$ & $1.5^{\mathrm{j}}$ & NT \\
\hline PFA/p-Fluoroamphetamine & $\mathrm{H}$ & $\mathrm{H}$ & $\mathrm{F}$ & $\mathrm{H}$ & $\mathrm{H}$ & & & $\mathrm{H}$ & $\mathrm{H}$ & $16^{j}$ & NT \\
\hline $\mathrm{POHA}$ & $\mathrm{H}$ & $\mathrm{H}$ & $\mathrm{OH}$ & $\mathrm{H}$ & $\mathrm{H}$ & & & $\mathrm{H}$ & $\mathrm{H}$ & $24.0^{k}$ & $N E^{k}$ \\
\hline (+)-Fenfluramine & $\mathrm{H}$ & $\mathrm{CF}_{3}$ & $\mathrm{H}$ & $\mathrm{H}$ & $\mathrm{H}$ & $\mathrm{CH}_{3}$ & $\mathrm{H}$ & $\mathrm{CH}_{2} \mathrm{CH}_{3}$ & $\mathrm{H}$ & $256^{\prime}$ & $800^{\prime}$ \\
\hline Fenfluramine & $\mathrm{H}$ & $\mathrm{CF}_{3}$ & $\mathrm{H}$ & $\mathrm{H}$ & $\mathrm{H}$ & & & $\mathrm{CH}_{2} \mathrm{CH}_{3}$ & $\mathrm{H}$ & $440^{\mathrm{m}}$ & $720^{\mathrm{m}}$ \\
\hline (-)-Fenfluramine & $\mathrm{H}$ & $\mathrm{CF}_{3}$ & $\mathrm{H}$ & $\mathrm{H}$ & $\mathrm{H}$ & $\mathrm{H}$ & $\mathrm{CH}_{3}$ & $\mathrm{CH}_{2} \mathrm{CH}_{3}$ & $\mathrm{H}$ & $115^{\prime}$ & $685^{\prime}$ \\
\hline (+)-Norfenfluramine & $\mathrm{H}$ & $\mathrm{CF}_{3}$ & $\mathrm{H}$ & $\mathrm{H}$ & $\mathrm{H}$ & $\mathrm{CH}_{3}$ & $\mathrm{H}$ & $\mathrm{H}$ & $\mathrm{H}$ & $36^{\prime}$ & $160^{\prime}$ \\
\hline
\end{tabular}

${ }^{a} / C_{50}$ and/or Ki are reported, depending on the reference considered. ${ }^{b}$ Chemical name and/or common acronym and/or common name is given. When not indicated the compound is the

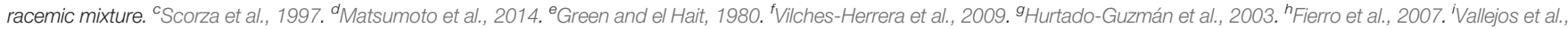

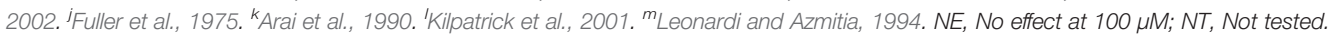

against MAO-A. Furthermore, when the $\beta$-keto substituent was replaced by a hydroxyl group, the compounds lost their activity on MAO-B while retaining MAOI-A inhibitory properties, although with lower potencies than their AMPH counterparts (Osorio-Olivares et al., 2004; Table 3).

\section{N-Substitution}

Relatively few amino group substituents have been studied in AMPH derivatives regarding their influence upon MAOI potency. In general terms, any $\mathrm{N}$-substitution leads to a decrease in the activity of the compound as a MAOI-A. Thus, the $N$-methyl derivatives of AMPH, MTA, $p$-methoxyAMPH
(PMA), and 3,4-methylenedioxyAMPH (MDA)- i.e. methamphetamine, NMMTA, PMMA, and MDMA respectively - have about one-third the inhibitory potency of their corresponding primary amine congeners (Scorza et al., 1997; Hurtado-Guzmán et al., 2003; Matsumoto et al., 2014; Santillo, 2014; Tables 1, 2, 4). In addition, enlargement of the amine substituent to $N$-ethyl, $N$ - $n$-propyl, or $N$-allyl seems to cause a further decrease in MAO-A affinity, correlated with the length of the substituent (Kilpatrick et al., 2001; HurtadoGuzmán et al., 2003; Santillo, 2014; Figure 7). Even larger substituents such as $N$-benzyl can lead to a complete loss of MAOI properties (Vilches-Herrera et al., 2009). Furthermore, 


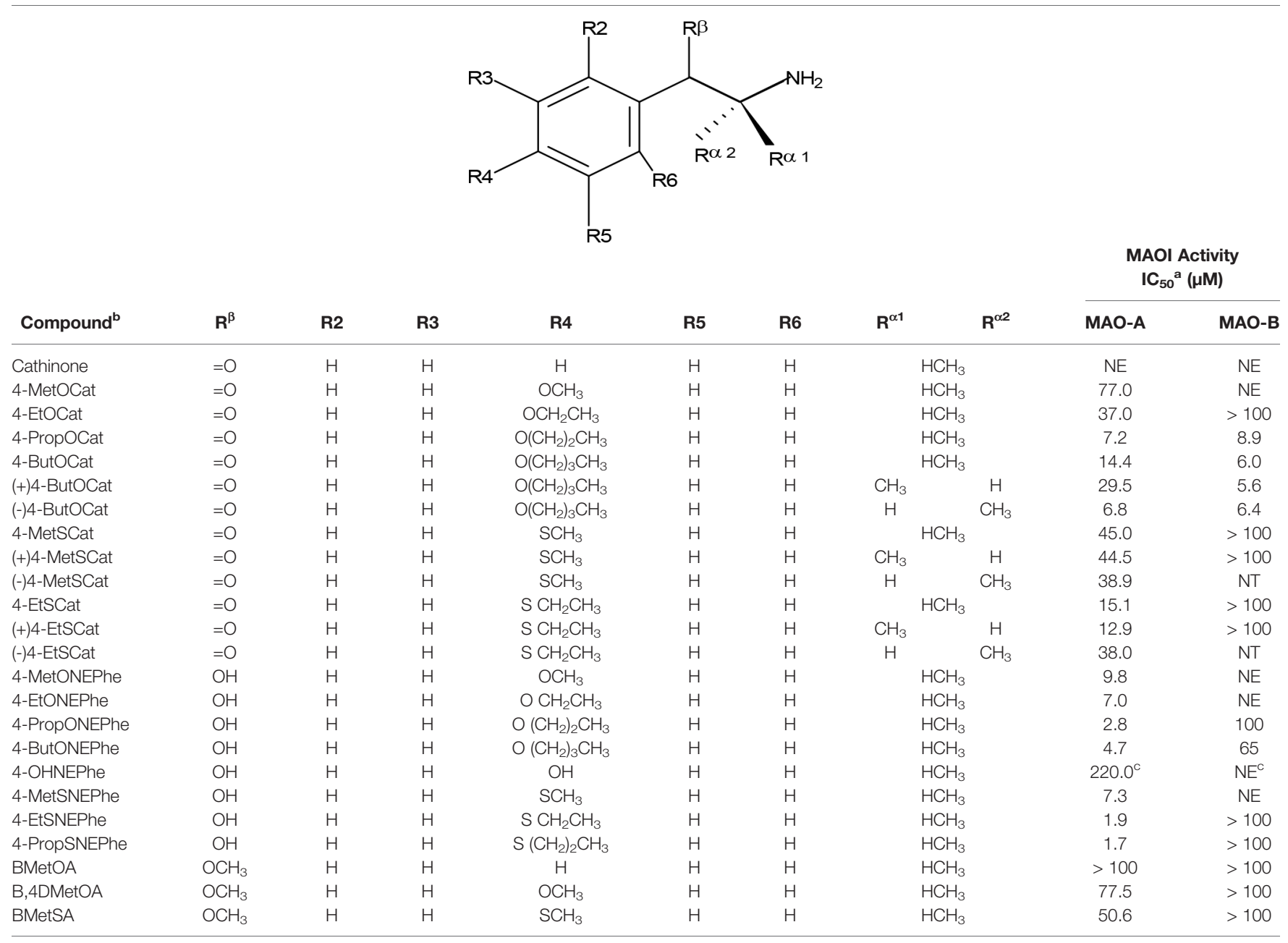

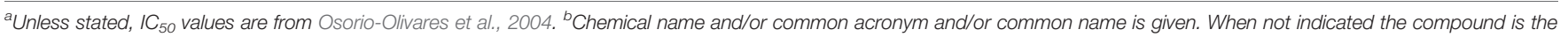
racemic mixture. ${ }^{\circ}$ Arai et al., 1990. NE, No effect at $100 \mu \mathrm{M}$; NT, Not tested.

tertiary amines (i.e. derivatives with a second group upon the amino moiety) are even less potent as MAOI-A than their secondary or primary analogues (Hurtado-Guzmán et al., 2003). These structure-activity relationships are very similar to those observed for AMPH derivatives regarding their interactions with DAT, NET, and SERT (Nichols, 1994), which agrees with a recent report that demonstrates a remarkable structural similarity between the ligand binding sites of MAOs and monoamine transporters (Nuñez-Vivanco et al., 2018).

\section{Aromatic Ring Substitution}

Even though some aliphatic MAO substrates and inhibitors have been identified (Yu et al., 1994; Yu et al., 1995; Kalgutkar et al., 2001), most studies have shown that the presence of an aromatic ring is essential for potent MAOI activity (Wouters, 1998; Kalgutkar et al., 2001; Tripathi et al., 2018; Dhiman et al., 2018). In the specific case of AMPH derivatives, docking simulations indicate that the benzene ring of these compounds binds to MAO-A mainly via interactions with $\pi$-systems of the catalytic site residues such as Y407, Y444 (both of which form part of the so called "aromatic cage"), Y69, F208, and/or F352 (Vallejos et al., 2005; Fierro et al., 2007; Vilches-Herrera et al., 2009; Fresqui et al., 2013; Vilches-Herrera et al., 2016; Figure 2). Accordingly, a handful of QSAR studies have shown that electronic features of the benzene ring (e.g. CHELPG atomic charges, HOMO energies) are the most important factors to determine the affinity of AMPH derivatives for MAO-A, and hence that their variation caused by substituents can greatly modulate their potency as enzyme inhibitors (Norinder et al, 1994; Vallejos et al., 2002; Fresqui et al., 2013). In this respect, the presence of a single substituent with electron-donor properties (e.g. alkoxy, alkylthio, alkylamino) at the para position of the aromatic ring seems to be the most influential substitution favoring potency toward MAO-A. Thus, MTA, PMA, and $p$ methylaminoAMPH (FLA727) are 20-50-fold more potent than the parent compound (Ask et al., 1985; Scorza et al., 1997; Tables 1, 2, Figure 8). The importance of the electron-donor character of the substituent at the para position is substantiated by the significant decrease of potency observed when the $p$-methylthio group is replaced by electron-withdrawing moieties such as $p$ - 
TABLE 4 | MAO inhibitory activity of AMPH derivatives polysubstituted in the aromatic ring

\begin{tabular}{|c|c|c|c|c|c|c|c|c|c|c|}
\hline \multirow[b]{2}{*}{ Compound $^{b}$} & \multirow[b]{2}{*}{$\mathbf{R 2}$} & \multirow[b]{2}{*}{ R3 } & \multirow[b]{2}{*}{ R4 } & \multirow[b]{2}{*}{ R5 } & \multirow[b]{2}{*}{ R6 } & \multirow[b]{2}{*}{$\alpha^{\alpha 1}$} & \multirow[b]{2}{*}{$\mathbf{R}^{\mathrm{N} 1}$} & \multirow[b]{2}{*}{$\mathrm{R}^{\mathrm{N} 2}$} & & \\
\hline & & & & & & & & & \multicolumn{2}{|c|}{$\begin{array}{c}\text { MAOI Activity } \\
\text { IC }_{50}(\mathrm{Ki})^{\mathrm{a}}(\mu \mathrm{M})\end{array}$} \\
\hline 2,4-DMA & $\mathrm{OCH}_{3}$ & $\mathrm{H}$ & $\mathrm{OCH}_{3}$ & $\mathrm{H}$ & $\mathrm{H}$ & & $\mathrm{H}$ & $\mathrm{H}$ & $0.6^{c}$ & $\mathrm{NE}^{\mathrm{C}}$ \\
\hline 3,4-DMA & $\mathrm{H}$ & $\mathrm{OCH}_{3}$ & $\mathrm{OCH}_{3}$ & $\mathrm{H}$ & $\mathrm{H}$ & & $\mathrm{H}$ & $\mathrm{H}$ & $20^{c}$ & $\mathrm{NE}^{\mathrm{C}}$ \\
\hline 2,5-DMA & $\mathrm{OCH}_{3}$ & $\mathrm{H}$ & $\mathrm{H}$ & $\mathrm{OCH}_{3}$ & $\mathrm{H}$ & & $\mathrm{H}$ & $\mathrm{H}$ & $>100^{f}$ & $N E^{f}$ \\
\hline 3,4,5-TMA & $\mathrm{H}$ & $\mathrm{OCH}_{3}$ & $\mathrm{OCH}_{3}$ & $\mathrm{OCH}_{3}$ & $\mathrm{H}$ & & $\mathrm{H}$ & $\mathrm{H}$ & $\mathrm{NE}^{\mathrm{c}} ; \mathrm{NI}^{\mathrm{d}}$ & $\mathrm{NE}^{\mathrm{c}} ; \mathrm{NI}^{\mathrm{d}}$ \\
\hline 2,4,5-TMA & $\mathrm{OCH}_{3}$ & $\mathrm{H}$ & $\mathrm{OCH}_{3}$ & $\mathrm{OCH}_{3}$ & $\mathrm{H}$ & & $\mathrm{H}$ & $\mathrm{H}$ & $\mathrm{NE}^{\mathrm{C}}$ & $\mathrm{NE}^{\mathrm{C}}$ \\
\hline 2,4,6-TMA & $\mathrm{OCH}_{3}$ & $\mathrm{H}$ & $\mathrm{OCH}_{3}$ & $\mathrm{H}$ & $\mathrm{OCH}_{3}$ & & $\mathrm{H}$ & $\mathrm{H}$ & $0.4^{\mathrm{e}}$ & $N E^{e}$ \\
\hline 2-Br-DMA & $\mathrm{Br}$ & $H$ & $\mathrm{OCH}_{3}$ & $\mathrm{OCH}_{3}$ & $\mathrm{H}$ & & $\mathrm{H}$ & $\mathrm{H}$ & $9.3^{c}$ & $\mathrm{NE}^{\mathrm{C}}$ \\
\hline 5-Br-DMA & $\mathrm{OCH}_{3}$ & $\mathrm{H}$ & $\mathrm{OCH}_{3}$ & $\mathrm{Br}$ & $\mathrm{H}$ & & $\mathrm{H}$ & $\mathrm{H}$ & $13.0^{c}$ & $\mathrm{NE}^{\mathrm{C}}$ \\
\hline $2-\mathrm{NO}_{2}-\mathrm{DMA}$ & $\mathrm{NO}_{2}$ & $\mathrm{H}$ & $\mathrm{OCH}_{3}$ & $\mathrm{OCH}_{3}$ & $\mathrm{H}$ & & $\mathrm{H}$ & $\mathrm{H}$ & $N E^{c}$ & $\mathrm{NE}^{\mathrm{C}}$ \\
\hline 6-Cl-DMA & $\mathrm{OCH}_{3}$ & $\mathrm{H}$ & $\mathrm{OCH}_{3}$ & $\mathrm{H}$ & $\mathrm{Cl}$ & & $\mathrm{H}$ & $\mathrm{H}$ & $0.07^{e}$ & $N E^{e}$ \\
\hline ALEPH-1 & $\mathrm{OCH}_{3}$ & $\mathrm{H}$ & $\mathrm{SCH}_{3}$ & $\mathrm{OCH}_{3}$ & $\mathrm{OCH}_{3}$ & & $\mathrm{H}$ & $\mathrm{H}$ & $5.1^{\mathrm{c}}$ & $\mathrm{NE}^{\mathrm{C}}$ \\
\hline ALEPH-2 & $\mathrm{OCH}_{3}$ & $\mathrm{H}$ & $\mathrm{SCH}_{2} \mathrm{CH}_{3}$ & $\mathrm{OCH}_{3}$ & $\mathrm{H}$ & & $\mathrm{H}$ & $\mathrm{H}$ & $3.2^{\mathrm{c}}$ & $\mathrm{NE}^{\mathrm{C}}$ \\
\hline 4-PrS-DMA & $\mathrm{OCH}_{3}$ & $\mathrm{H}$ & $\mathrm{S}\left(\mathrm{CH}_{2}\right)_{2} \mathrm{CH}_{3}$ & $\mathrm{OCH}_{3}$ & $\mathrm{H}$ & & $\mathrm{H}$ & $\mathrm{H}$ & $2.4^{e}$ & $\mathrm{NE}^{\mathrm{e}}$ \\
\hline 4-BuS-DMA & $\mathrm{OCH}_{3}$ & $\mathrm{H}$ & $\mathrm{S}\left(\mathrm{CH}_{2}\right)_{3} \mathrm{CH}_{3}$ & $\mathrm{OCH}_{3}$ & $\mathrm{H}$ & & $\mathrm{H}$ & $\mathrm{H}$ & $2.9^{e}$ & $N E^{e}$ \\
\hline 4-PentS-DMA & $\mathrm{OCH}_{3}$ & $\mathrm{H}$ & $\mathrm{S}\left(\mathrm{CH}_{2}\right)_{4} \mathrm{CH}_{3}$ & $\mathrm{OCH}_{3}$ & $\mathrm{H}$ & & $\mathrm{H}$ & $\mathrm{H}$ & $14.3^{\mathrm{e}}$ & $N E^{e}$ \\
\hline 2,5-DM-MTAB & $\mathrm{OCH}_{3}$ & $\mathrm{H}$ & $\mathrm{SCH}_{3}$ & $\mathrm{OCH}_{3}$ & $\mathrm{H}$ & & $\mathrm{H}$ & $\mathrm{H}$ & $30.9^{e}$ & $N E^{e}$ \\
\hline 2,5-DM-ETAB & $\mathrm{OCH}_{3}$ & $\mathrm{H}$ & $\mathrm{SCH}_{2} \mathrm{CH}_{3}$ & $\mathrm{OCH}_{3}$ & $\mathrm{H}$ & & $\mathrm{H}$ & $\mathrm{H}$ & $11.8^{\mathrm{e}}$ & $\mathrm{NE}^{\mathrm{e}}$ \\
\hline 2,6-DM-MTA & $\mathrm{OCH}_{3}$ & $\mathrm{H}$ & $\mathrm{SCH}_{3}$ & $\mathrm{H}$ & $\mathrm{OCH}_{3}$ & & $\mathrm{H}$ & $\mathrm{H}$ & $0.30^{\mathrm{e}}$ & $N E^{e}$ \\
\hline 2,6-DM-ETA & $\mathrm{OCH}_{3}$ & $\mathrm{H}$ & $\mathrm{SCH}_{2} \mathrm{CH}_{3}$ & $\mathrm{H}$ & $\mathrm{OCH}_{3}$ & & $\mathrm{H}$ & $\mathrm{H}$ & $0.08^{\mathrm{e}}$ & $N E^{e}$ \\
\hline 4-ESO-2,5-DMA & $\mathrm{OCH}_{3}$ & $\mathrm{H}$ & $\mathrm{SOCH}_{3}$ & $\mathrm{OCH}_{3}$ & $\mathrm{H}$ & & $\mathrm{H}$ & $\mathrm{H}$ & $>100^{f}$ & NT \\
\hline 4-ESO2-2,5-DMA & $\mathrm{OCH}_{3}$ & $\mathrm{H}$ & $\mathrm{SO}_{2} \mathrm{CH}_{3}$ & $\mathrm{OCH}_{3}$ & $\mathrm{H}$ & & $\mathrm{H}$ & $\mathrm{H}$ & $N E^{f}$ & NT \\
\hline DOM & $\mathrm{OCH}_{3}$ & $\mathrm{H}$ & $\mathrm{CH}_{3}$ & $\mathrm{OCH}_{3}$ & $\mathrm{H}$ & & $\mathrm{H}$ & $\mathrm{H}$ & $24.0^{\mathrm{C}}$ & $\mathrm{NE}^{\mathrm{C}}$ \\
\hline DOI & $\mathrm{OCH}_{3}$ & $\mathrm{H}$ & I & $\mathrm{OCH}_{3}$ & $\mathrm{H}$ & & $\mathrm{H}$ & $\mathrm{H}$ & $24^{\mathrm{c}} ; 37^{\mathrm{d}}$ & $\mathrm{NE}^{\mathrm{C}}$ \\
\hline DOB & $\mathrm{OCH}_{3}$ & $\mathrm{H}$ & $\mathrm{Br}$ & $\mathrm{OCH}_{3}$ & $\mathrm{H}$ & & $\mathrm{H}$ & $\mathrm{H}$ & $100^{c}$ & $\mathrm{NE}^{\mathrm{C}}$ \\
\hline DON & $\mathrm{OCH}_{3}$ & $\mathrm{H}$ & $\mathrm{NO}_{2}$ & $\mathrm{OCH}_{3}$ & $\mathrm{H}$ & & $\mathrm{H}$ & $\mathrm{H}$ & $\mathrm{NE}^{\mathrm{C}}$ & $\mathrm{NE}^{\mathrm{C}}$ \\
\hline DOTFM & $\mathrm{OCH}_{3}$ & $\mathrm{H}$ & $\mathrm{CF}_{3}$ & $\mathrm{OCH}_{3}$ & $\mathrm{H}$ & & $\mathrm{H}$ & $\mathrm{H}$ & $N E^{c}$ & $\mathrm{NE}^{\mathrm{C}}$ \\
\hline MDA & $\mathrm{H}$ & & $-\mathrm{O}-\mathrm{CH}_{2}$ & $\mathrm{H}$ & $\mathrm{H}$ & & $\mathrm{H}$ & $\mathrm{H}$ & $9.3^{\mathrm{C}}\left(8.5^{\mathrm{g}}\right)$ & $\mathrm{NE}^{\mathrm{C}}$ \\
\hline 2Br-MDA & $\mathrm{Br}$ & $\mathrm{H}$ & $\mathrm{CH}_{2}-\mathrm{C}$ & & $\mathrm{H}$ & & $\mathrm{H}$ & $\mathrm{H}$ & $13.0^{c}$ & $64.0^{C}$ \\
\hline 2Cl-MDA & $\mathrm{Cl}$ & $\mathrm{H}$ & $\mathrm{CH}_{2}-\mathrm{C}$ & & $\mathrm{H}$ & & $\mathrm{H}$ & $\mathrm{H}$ & $6.3^{c}$ & $38.0^{C}$ \\
\hline $2 \mathrm{NO}_{2}-\mathrm{MDA}$ & $\mathrm{NO}_{2}$ & $\mathrm{H}$ & $\mathrm{CH}_{2}-\mathrm{C}$ & & $\mathrm{H}$ & & $\mathrm{H}$ & $\mathrm{H}$ & $\mathrm{NE}^{\mathrm{C}}$ & $N E^{C}$ \\
\hline MDMA & $\mathrm{H}$ & & $-\mathrm{O}-\mathrm{CH}_{2}$ & $\mathrm{H}$ & $\mathrm{H}$ & & $\mathrm{CH}_{3}$ & $\mathrm{H}$ & $30^{c}\left(24.7^{g}\right)$ & $\mathrm{NE}^{\mathrm{C}}$ \\
\hline (+)-MDMA & $\mathrm{H}$ & & $-\mathrm{O}-\mathrm{CH}_{2}$ & $\mathrm{H}$ & $\mathrm{H}$ & & $\mathrm{CH}_{3}$ & $\mathrm{H}$ & $44^{h}\left(22^{h}\right)$ & $370^{h}$ \\
\hline (-)-MDMA & $\mathrm{H}$ & & $-\mathrm{O}-\mathrm{CH}_{2}$ & $\mathrm{H}$ & $\mathrm{H}$ & & $\mathrm{CH}_{3}$ & $\mathrm{H}$ & $56^{h}\left(28.3^{h}\right)$ & $378^{h}$ \\
\hline
\end{tabular}

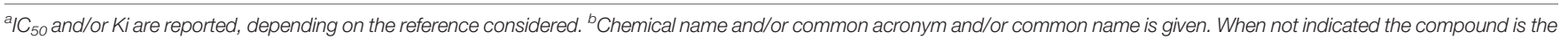

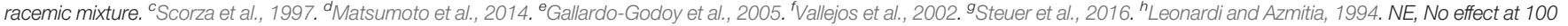
$\mu \mathrm{M} ;$ NI, No inhibition at $200 \mu \mathrm{M} ;$ NT, Not tested.

methylsulfoxy or $p$-methylsulfonyl (i.e. MSOA or MSO2A, respectively; Vallejos et al., 2002; Table 2, Figure 8). It is worth pointing out that para-halogenated AMPHs (PCA, PBA, and PFA) are also fairly potent MAOI-A, with their potencies negatively correlated with their electron-withdrawing character, that is, PBA and PCA being about 7- and 4-fold more potent, and PFA being slightly less potent than AMPH (Fuller et al., 1975; Scorza et al., 1997; Table 2, Figure 8). Modestly increasing the size of the para-sulfur/oxygen substituent with linear aliphatic chains (i.e. ethyl-, propyl-, butyl-) leads to an increase in potency, while larger or branched substituents generate less potent compounds (Scorza et al., 1997; Gallardo-Godoy et al., 2005; Fierro et al., 2007; Vilches-Herrera et al., 2009 and VilchesHerrera et al., 2016; Figure 9). It has been suggested (Fierro et al.,
2007) that the increase in potency might be related to steric parameters of the para substituent (van der Waals volume and/ or Taft steric parameter $E_{s}$ ) which would be optimal for alkyl chains containing up to four carbon atoms. Longer chains, however, might oblige the compound to adopt a more folded conformation, which would disfavor their interaction with the residues at the binding site. Interestingly, cycloalkyl analogues of pentylthioAMPH and hexylthioAMPH showed higher rat MAOI-A potencies than their $n$-alkyl counterparts, which was attributed to a better fit within the binding site due to the entropic advantage conferred by their "precoiled" conformations (Vilches-Herrera et al., 2016; Figure 9). In contrast, the presence of substituents at the ortho or meta positions in para-unsubstituted compounds had no effect or 
TABLE 5 | MAO inhibitory activity of some AMPH derivatives containing aromatic systems larger than benzene.

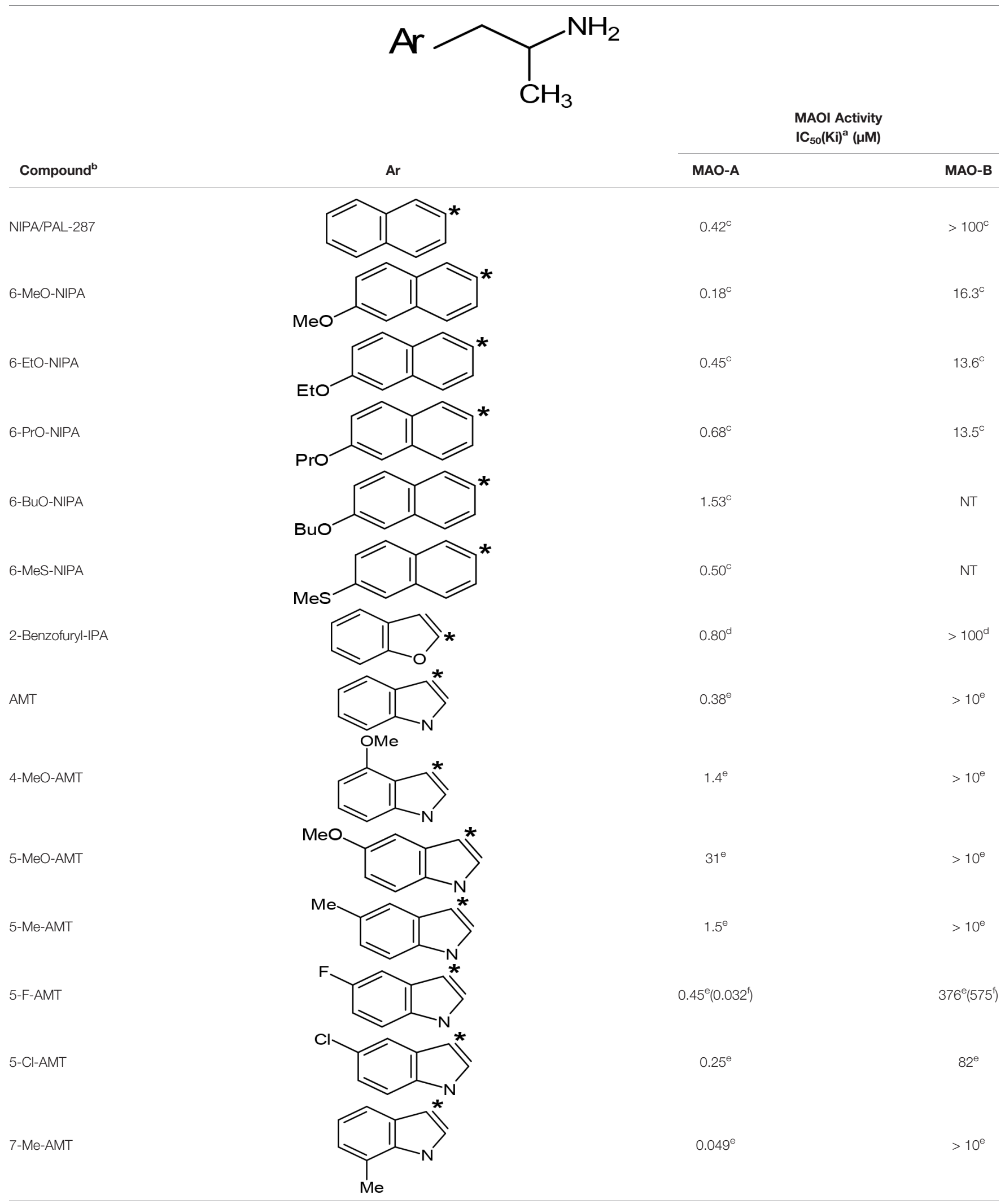

${ }^{a} C_{50}$ and/or $K_{i}$ are reported, depending on the reference considered. ${ }^{b}$ Chemical name and/or common acronym and/or common name is given. ${ }^{c}$ Vilches-Herrera et al., 2009. ${ }^{d}$ Vallejos et al., 2005. 'Wagmann et al., 2017. ' Kinemuchi et al., 1988. NT, Not tested. Ar, Aromatic ring. *: This symbol denotes where, in the aromatic ring, the aliphatic side chain is linked. Me, Methyl; Et, Ethyl; Pr, Propyl; Bu, Butyl. 

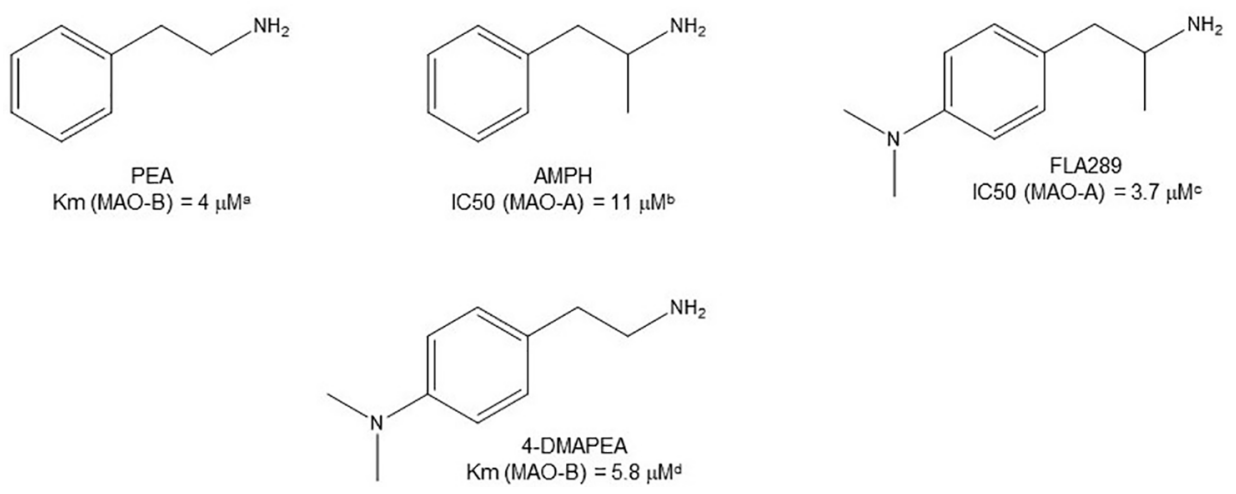

FIGURE 3 | Chemical structures and MAO parameters of some phenethylamine/AMPH derivative pairs. ${ }^{a}$ Youdim et al., 2006. 'bScorza et al., 1997. 'Reyes-Parada et al., 1994b. 'Reyes-Parada et al., 1994a.

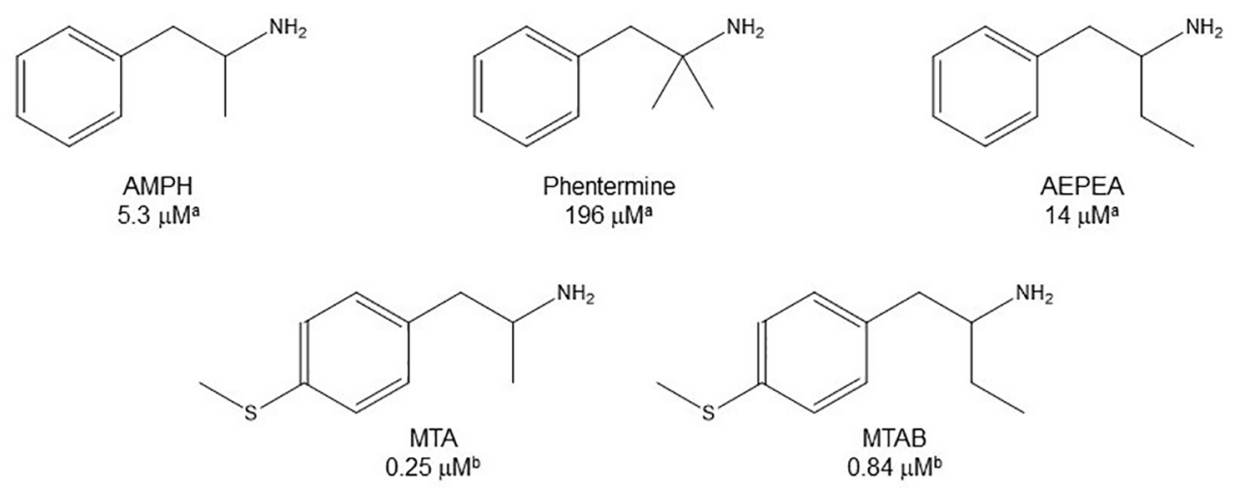

FIGURE 4 | Chemical structures and MAO-A IC 50 of some $\alpha$-substituted AMPH derivatives. aSantillo, 2014. ' bHurtado-Guzmán et al., 2003.

led to a decrease of the potency as MAOI-A as compared with AMPH (Green and el Hait, 1980; Scorza et al., 1997; Kilpatrick et al., 2001; Tables 2 and 4). Moreover, the addition of bulky groups adjacent to the para-substituted position induces a decrease in potency. Thus, the presence of one or two substituents at the meta position(s) of PMA produce compounds that are respectively 60-fold less potent or completely inactive (Scorza et al., 1997; Figure 10). Nonetheless, the introduction of substituents at more distant positions has less detrimental effects on potency, and even in some cases a marked increase of activity (and selectivity) has been reported. Thus, the addition of a methyl group at the ortho position of $p$-dimethylaminoAMPH (FLA289) to yield FLA336 produced a slight increase in potency, which was more evident (along with a remarkable increase in MAO-A/B selectivity) in the case of amiflamine, the $(S)-(+)$-isomer of FLA336 (Ask et al., 1985; Table 1). Indeed, the introduction of one or two halogen atoms $(\mathrm{Cl}$ or $\mathrm{Br})$ at the ortho position(s), generates some of the most potent MAOI-A AMPH derivatives described until now (Ask et al., 1985; Gallardo-Godoy et al., 2005; Tables 1 and 4).

The expansion of the aromatic ring of AMPH is an additional modification that has yielded interesting results and potent and selective MAOIs-A (Table 5). This could have been anticipated considering that: a) the structure of 5-HT, one of the natural substrates of MAO-A, contains an indolyl moiety instead a simple benzene ring and; b) the electron-richness of the aromatic ring seems to be an important molecular determinant for MAO-A affinity (Vallejos et al., 2002), and therefore aromatic systems larger than benzene might establish stronger $\pi$-type interactions with aromatic and non-aromatic residues present in the active site of the protein. Accordingly, for example 2-naphthylisopropylamine (NIPA, also known as PAL-287; Rothman et al., 2005) and its methoxylated or methylthio derivatives (Vilches-Herrera et al., 2009), 2-benzofurylisopropylamine (Vallejos et al., 2005) or several $\alpha$-methyltryptamine derivatives (Kinemuchi et al., 1988; Wagmann et al., 2017) have been shown to be highly selective 


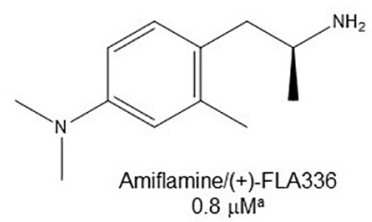<smiles>CSc1ccc(C[C@@H](C)N)cc1</smiles><smiles>CSc1ccc(C[C@@H](C)N)cc1</smiles>

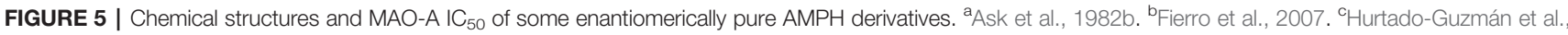
2003<smiles>CC(N)Cc1ccccc1</smiles>

AMPH

IC50 (MAO-A) $=11 \mu \mathrm{M}^{\mathrm{a}}$<smiles>CC(N)C(=O)c1ccccc1</smiles>

Cathinone Not effect at $100 \mu M^{b}$<smiles>CSc1ccc(CC(C)N)cc1</smiles>

MTA $0.25 \mu \mathrm{M}^{\mathrm{c}}$<smiles>CSc1ccc(C(=O)C(C)N)cc1</smiles>

FIGURE 6 | Chemical structures and MAO-A IC 50 of some $\beta$-keto substituted AMPH derivatives. a'Scorza et al., 1997. ' ${ }^{\circ}$ Osorio-Olivares et al., 2004. ${ }^{\text {CHurtado- }}$ Guzmán et al., 2003.

MAOI-A, much more potent than AMPH (Table 5). Both electronic and steric factors have been invoked to explain the higher activity of AMPH derivatives containing aromatic rings larger than benzene (Vallejos et al., 2005; Vilches-Herrera et al., 2009). Thus, $\pi$ systems with an increased electron-donating capacity, softer and/or more polarizable as compared with benzene, might favor charge-transfer (Vallejos et al., 2002) and/ or $\pi$-stacking interactions (Fresqui et al., 2013) with aromatic fragments in the active site. In addition, docking simulations have shown that AMPH derivatives containing large aromatic rings, can establish interactions not only with the aromatic residues forming the so-called aromatic cage (i.e. Y407 and Y444), but also with aminoacids located more distantly, such as F208, Y69, and F352 (Vallejos et al., 2005; Vilches-Herrera et al., 2009). Therefore, the higher potency of AMPH derivatives containing condensed aromatic systems could be explained by an increased probability of establishing dispersive short length interactions and also a greater number of interactions.

\section{SUMMARY OF STRUCTURE-ACTIVITY RELATIONSHIPS AND IMPLICATIONS OF MAOI PROPERTIES FOR THE OVERALL PHARMACOLOGY OF AMPH DERIVATIVES}

Potent, selective, and competitive MAO-A inhibitory properties are found in many AMPH derivatives. This is likely due to the structural similarity of this type of compounds with physiological 


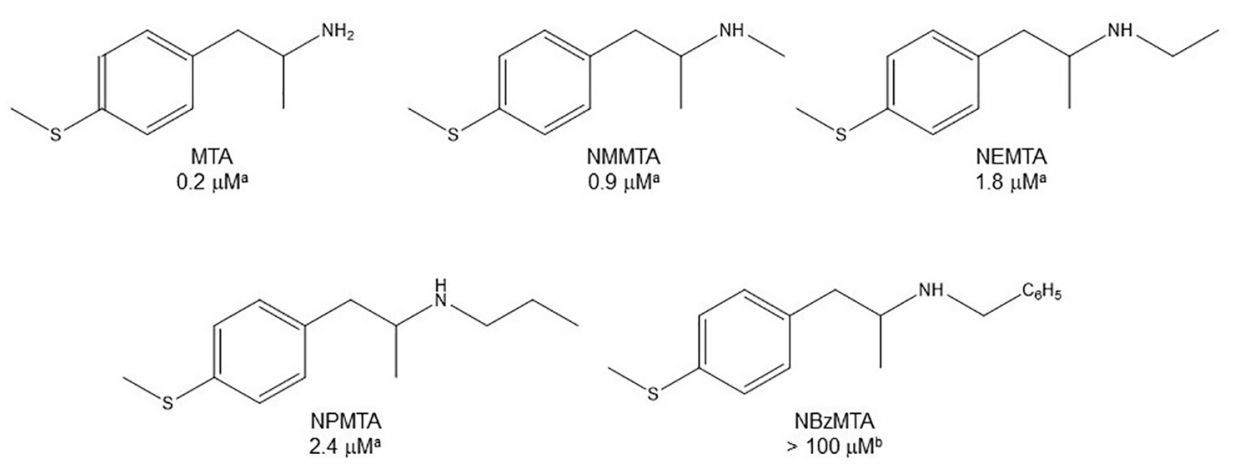

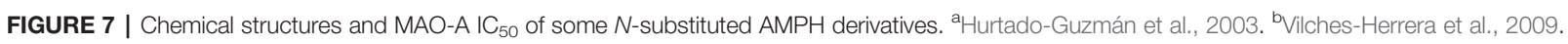<smiles>CSc1ccc(CC(C)N)cc1</smiles><smiles>COc1ccc(CC(C)N)cc1</smiles><smiles>CNc1ccc(CC(C)N)cc1</smiles><smiles>CC(N)C(=O)c1ccccc1</smiles><smiles>CSc1ccc(C(=O)C(C)N)cc1</smiles><smiles>CC(N)Cc1ccc(S(C)=O)cc1</smiles><smiles>CC(N)Cc1ccc(S(C)(=O)=O)cc1</smiles><smiles>CC(N)Cc1ccc(F)cc1</smiles><smiles>CC(N)Cc1ccc(Cl)cc1</smiles><smiles>CC(N)Cc1ccc(Br)cc1</smiles>

FIGURE 8 | Chemical structures and MAO-A IC 50 of some p-substituted AMPH derivatives. ${ }^{a}$ Hurtado-Guzmán et al., 2003. ' ${ }^{\text {S }}$ corza et al., 1997. ${ }^{\circ}$ Reyes-Parada et al., 1994a. 'Osorio-Olivares et al., 2004. 'Vallejos et al., 2002. fFuller et al., 1975.

substrates, which allows AMPH derivatives to occupy, and consequently block the access of any substrate into the active site of the enzyme.

Although not extensively studied, in general the introduction of diverse substituents on either the amino group or the side chain of the basic AMPH skeleton leads to compounds with lower affinity as compared with the parent counterparts. Furthermore, several studies have shown that $(S)-(+)-\mathrm{AMPH}$ derivatives are the eutomers for MAO inhibition. Besides, as aromatic interactions in the active site of the enzyme seem to be critical for inhibition, substituents at this portion of the AMPH structure greatly modulate its potency. In general, electron- donor substituents at the para position of the aromatic ring generate potent MAOI-A, while substituents adjacent to this position decrease activity. In addition, replacement of the benzene ring by larger $\pi$ systems exhibiting an increased electron-donating capacity, generates compounds with higher MAOI potency.

Even though MAO inhibition has been demonstrated for several AMPH derivatives, this is often considered not relevant for their global effect, since their affinity for MAO is usually weak compared to affinity for their main pharmacological targets (i.e. monoamine transporters or receptors). This is most likely true in the case of compounds such as the anti-obesity agents 
<smiles>CCCSc1ccc(CC(C)N)cc1</smiles><smiles>CC(Cc1ccc(SC(C)C)cc1)=NSc1ccc(CC(C)CSc2ccc(CC(C)N)cc2)cc1</smiles><smiles>CC(N)Cc1ccc(SC2CCCC2)cc1</smiles><smiles>CC(N)Cc1ccc(SC2CCCCC2)cc1</smiles>

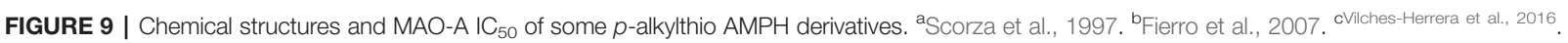<smiles>COc1ccc(CC(C)N)cc1</smiles><smiles>COc1ccc(CC(C)N)cc1OC</smiles><smiles>COc1cc(CC(C)N)cc(OC)c1OC</smiles>

FIGURE 10 | Chemical structures and MAO-A IC 50 of some $p$-methoxy AMPH derivatives. IC 50 values are from Scorza et al., 1997. NE, No effect.

phentermine and fenfluramine (Kilpatrick et al., 2001; Nandigama et al., 2002), or the hallucinogenic drugs DOI and DOB (Nichols, 2018), whose ability either to evoke monoamine release or to activate 5-HT receptors exceeds by several orders of magnitude their potency as MAOIs. However, in the case of monoamine releasing agents such as PMA or MTA, their potency upon their main protein targets (i.e. SERT and DAT) is remarkably similar to that reported for MAO-A (Green and el Hait, 1980; Nichols et al., 1993; Scorza et al., 1997; Callaghan et al., 2005; Gobbi et al., 2008; Sotomayor-Zárate et al., 2012; Matsumoto et al., 2014). Thus, one may assume that the cases of severe toxicity reported after recreational use of these drugs (e.g. Elliott, 2000; De Letter et al., 2001; Martin, 2001; Lamberth et al., 2008), which resemble "serotonin syndrome" symptoms (Lapoint et al., 2013), are related to a sustained increase of synaptic 5-HT and DA resulting from both monoamine reverse transport and MAO-A inhibition. Furthermore, as many AMPH derivatives are monoamine transporter substrates (Simmler et al., 2013; Sitte and Freissmuth, 2015), even in those cases in which relatively weak MAOI activity is demonstrated, these drugs might be concentrated in presynaptic nerve terminals or glial cells, and some enzyme inhibition could occur (Heal et al., 2013). In this sense, accumulation and pronounced inhibition of
MAO in monoaminergic neurons has been consistently reported for amiflamine and its analogues (Ask et al., 1983; Ask et al., 1985; Ask et al., 1986; Ask and Ross, 1987). In addition, especially after relatively prolonged use, antidepressant and/or anxiolytic effects derived from MAO-A inhibition might influence the global pharmacological effect of AMPH derivatives. Thus, for example, considering the high overlap between depression and drug abuse (Markou et al., 1998; Bruijnzeel et al., 2004), it is enticing to suggest that at least part of the antiaddictive potential of PAL-287 (NIPA; Table 5), which has been attributed to its DA and 5-HT releasing properties (Rothman et al., 2005; Rothman et al., 2007; Rothman et al., 2008), might be related also to its MAOI-A activity (Vilches-Herrera et al., 2009). Moreover, subtle differences in the subjective experience generated by hallucinogenic AMPH derivatives such as DOI or DOB as compared with sulfur containing analogues (Shulgin and Shulgin, 1991), might be associated with the much more pronounced MAOI-A activity of the latter (Table 4, Scorza et al., 1997; Gallardo-Godoy et al., 2005). Thus, these and other examples highlight the notion that MAOI properties should be considered when assessing the overall pharmacology of AMPH derivatives. 


\section{CONCLUDING REMARKS}

Although many AMPH derivatives have been tested as MAOI, the structural diversity of such compounds is relatively limited. This calls for a broader exploration of the chemical space around the parent scaffold in the search of compounds with novel properties, in which MAOI properties might or might not be pursued. As known and unknown AMPH derivatives are usually attractive for illicit purposes (production, marketing, and/or consumption), it seems very relevant to evaluate in every case the possible MAOI activity of these drugs, since it may convey dangerous consequences for uninformed users.

Regarding the mechanism of enzyme inhibition, insofar as a crystal structure of MAO in complex with some AMPH derivative is not available, molecular simulation appears as one of the most reliable tools to study this issue. Nevertheless, most of current information has been obtained through docking studies, without resorting to molecular dynamics simulations that consume much more computer time, and therefore models generated still require a further validation. In addition, several reports indicate that MAOI profiles differ if enzymes from human or other species are used (not only for AMPH derivatives). Hence, inferences regarding possible effects in humans should be most cautious when data are obtained initially in animal models. Moreover, in comparison to recent characterizations of the monoamine transporter and receptor interactions of amphetamines (Simmler et al., 2013; Luethi and Liechti, 2018), the MAO inhibiting properties have not been investigated using the same assays across a larger range of

\section{REFERENCES}

Angoa-Pérez, M., Anneken, J. H., and Kuhn, D. M. (2017). Neurotoxicology of Synthetic Cathinone Analogs. Curr. Top. Behav. Neurosci. 32, 209-230. doi: 10.1007/7854_2016_21

Arai, Y., Kim, S. K., Kinemuchi, H., Tadano, T., Satoh, S. E., Satoh, N., et al. (1990). Inhibition of brain type A monoamine oxidase and 5-hydroxytryptamine uptake by two amphetamine metabolites, p-hydroxyamphetamine and phydroxynorephedrine. J. Neurochem. 55, 403-408. doi: 10.1111/j.14714159.1990.tb04151.x

Ask, A. L., and Ross, S. B. (1987). Inhibition of 5-hydroxytryptamine accumulation and deamination by substituted phenylalkylamines in hypothalamic synaptosomes from normal and reserpine-pretreated rats. Naunyn Schmiedebergs Arch. Pharmacol. 336, 591-596. doi: 10.1007/bf00165748

Ask, A. L., Hellström, W., Norrman, S., Ögren, S. O., and Ross, S. B. (1982a). Selective inhibition of the A form of monoamine oxidase by 4 dimethylamino-alpha-methylphenylalkylamine derivatives in the rat. Neuropharmacology21, 299-308. doi: 10.1016/0028-3908(82)90092-2

Ask, A. L., Högberg, K., Schmidt, L., Kiessling, H., and Ross, S. B. (1982b). (+)-4Dimethylamino-2,alpha-dimethylphenethylamine (FLA 336(+)), a selective inhibitor of the A form of monoamine oxidase in the rat brain. Biochem. Pharmacol. 31, 1401-1406. doi: 10.1016/0006-2952(82)90035-1

Ask, A. L., Fagervall, I., and Ross, S. B. (1983). Selective inhibition of monoamine oxidase in monoaminergic neurons in the rat brain. Naunyn-Schmiedebergs Arch. Pharmacol. 324, 79-87. doi: 10.1007/bf00497011

Ask, A. L., Fagervall, I., Florvall, L., Ross, S. B., and Ytterborn, S. (1985). Inhibition of monoamine oxidase in 5-hydroxytryptaminergic neurones by substituted paminophenylalkylamines. Br. J. Pharmacol. 85, 683-690. doi: 10.1111/j.14765381.1985.tb10564.x substances. Therefore, comparative analyses should be done cautiously when considering results obtained under different experimental conditions

Beyond these considerations, in our view it is clear that AMPH derivatives can act as MAOI and that this activity should be taken into account when analyzing the overall pharmacodynamics of these structurally versatile compounds.

\section{AUTHOR CONTRIBUTIONS}

All authors equally contributed to the writing of this manuscript.

\section{FUNDING}

The constant support of FONDECYT, in particular grants 1170662 (MR-P), 1150615 (PI-V), and 1150868 (BKC), is gratefully acknowledged.

\section{ACKNOWLEDGMENTS}

The authors are grateful to all the colleagues who have contributed over the years to the studies carried out in our laboratories on the MAOI properties of AMPH derivatives. We also thank Dr. Patricia Möller-Acuña for her valuable help in doing measurements and preparing Figure 2.

Ask, A. L., Fagervall, I., Florvall, L., Ross, S. B., and Ytterborn, S. (1986). Selective inhibition of monoamine oxidase by p-aminosubstituted phenylalkylamines in catecholaminergic neurones. Neuropharmacology 25, 33-40. doi: 10.1016/ 0028-3908(86)90055-9

Barbosa, D. J., Capela, J. P., Feio-Azevedo, R., Teixeira-Gomes, A., Bastos, Mde, L., and Carvalho, F. (2015). Mitochondria: key players in the neurotoxic effects of amphetamines. Arch. Toxicol. 89, 1695-1725. doi: 10.1007/s00204-015-1478-9

Biel., J. H., and Bopp, B. A. (1978). "Amphetamines: structure-activity relationships," in Handbook of Psychopharmacology. Eds. L. L. Iversen, S. D. Iversen and S. H. Snyder (New York, NY: Stimulants. Plenum), 1-40.

Binda, C., Newton-Vinson, P., Hubálek, F., Edmondson, D. E., and Mattevi, A. (2002). Structure of human monoamine oxidase B, a drug target for the treatment of neurological disorders. Nat. Struct. Biol. 9, 22-26. doi: 10.1038/nsb732

Binda, C., Li, M., Hubálek, F., Restelli, N., Edmondson, D. E. , and Mattevi, A. (2003). Insights into the mode of inhibition of human mitochondrial monoamine oxidase B from high-resolution crystal structures. Proc. Natl. Acad. Sci. U. S. A. 100 8755-9759. doi: 10.1073/pnas.1633804100

Binda, C., Hubálek, F., Li, M., Herzig, Y., Sterling, J., Edmondson, D. E., et al. (2004). Crystal structures of monoamine oxidase B in complex with four inhibitors of the N-propargylamino-indan class. J. Med. Chem. 47, 1767-1774. doi: $10.1021 / j m 031087 \mathrm{c}$

Binda, C., Mattevi, A., and Edmondson, D. E. (2011). Structural properties of human monoamine oxidases A, and B. Int. Rev. Neurobiol. 100, 1-11. doi: 10.1016/B978-0-12-386467-3.00001-7

Bruijnzeel., A. W., Repetto, M., and Gold, M. S. (2004). Neurobiological mechanisms in addictive and psychiatric disorders. Psychiatr. Clin. North Am. 27, 661-674. doi: 10.1016/j.psc.2004.06.005

Callaghan, P. D., Irvine, R. J., and Daws, L. C. (2005). Differences in the in vivo dynamics of neurotransmitter release and serotonin uptake after acute para- 
methoxyamphetamine and 3,4-methylenedioxymethamphetamine revealed by chronoamperometry. Neurochem. Int. 47, 350-361. doi: 10.1016/ j.neuint.2005.04.026

Cesura, A. M., and Pletscher, A. (1992). The new generation of monoamine oxidase inhibitors. Prog. Drug Res. 38, 171-297. doi: 10.1007/978-3-0348-71419_3

Dauvilliers, Y., and Barateau, L. (2017). Narcolepsy and Other Central Hypersomnias. Continuum (Minneapolis, MN). Sleep Neurol. 23:1, 989-1004. doi: 10.1212/CON.0000000000000492

De Colibus, L., Li, M., Binda, C., Lustig, A., Edmondson, D. E., and Mattevi, A. (2005). Three-dimensional structure of human monoamine oxidase A (MAO A): Relation to the structures of rat MAO A and human MAO B. Proc. Natl. Acad. Sci. U. S. A. 102, 12684-12689. doi: 10.1073/pnas.0505975102

De Letter, E. A., Coopman, V. A., Cordonnier, J. A., and Piette, M. H. (2001). One fatal and seven non-fatal cases of 4-methylthioamphetamine (4-MTA) intoxication: clinico-pathological findings. Int. J. Legal Med. 114, 352-356. doi: $10.1007 /$ s004140100204

Dhiman, P., Malik, N., and Khatkar, A. (2017). Docking-Related Survey on Natural-Product-Based New Monoamine Oxidase Inhibitors and Their Therapeutic Potential. Comb. Chem. High Throughput Screen. 20, 474-491. doi: 10.2174/1386207320666170414102814

Dhiman, P., Malik, N., and Khatkar, A. (2018). 3D-QSAR and in-silico Studies of Natural Products and Related Derivatives as Monoamine Oxidase Inhibitors. Curr. Neuropharmacol. 16, 881-900. doi: 10.2174/1570159X1566617 1128143650

Edeleano, L. (1887). Ueber einige derivate der phenylmethacrylsäure und der phenylisobuttersaure. Ber. Deut. Chem. Ges. 20, 616-622. doi: 10.1002/ cber. 188702001142

Edmondson, D. E., Binda, C., and Mattevi, A. (2007). Structural insights into the mechanism of amine oxidation by monoamine oxidases A, and B. Arch. Biochem. Biophys. 464, 269-276. doi: 10.1016/j.abb.2007.05.006

Edwards, D. J. (1978). Phenylethanolamine is a specific substrate for type B monoamine oxidase. Life Sci. 23, 1201-1207. doi: 10.1016/0024-3205(78) 90355-7

Elliott, S. P. (2000). Fatal poisoning with a new phenylethylamine: 4methylthioamphetamine (4-MTA). J. Anal. Toxicol. 24, 85-89. doi: 10.1093/ jat $/ 24.2 .85$

Fagervall, I., Kelder, D., and Ross, S. B. (1988). Selective inhibition by 4,alphadimethyl-m-tyramine (H77/77) and 4-methyl-alpha-ethyl-m-tyramine (H75/ 12) of the monoamine oxidase within serotonergic and noradrenergic neurons in the rat brain. Naunyn-Schmiedebergs Arch. Pharmacol. 338, 143-147. doi: $10.1007 / \mathrm{bf} 00174862$

Feenstra, M. G., van der Velden, T., Dijkstra, D., Hommes, O. R., and Horn, A. S. (1983). Inhibition of rat brain monoamine oxidase type A by 2 -aminotetralin and tetrahydroisoquinoline analogues of dopamine. Pharm. Weekbl. Sci. 5, 131-133. doi: 10.1007/bf01961468

Ferino, G., Vilar, S., Matos, M. J., Uriarte, E., and Cadoni, E. (2012). Monoamine oxidase inhibitors: ten years of docking studies. Curr. Top. Med. Chem. 12, 2145-2162. doi: 10.2174/156802612805220048

Fierro, A., Osorio-Olivares, M., Cassels, B. K., Edmondson, D. E., Sepúlveda-Boza, S., and Reyes-Parada, M. (2007). Human and rat monoamine oxidase-A are differentially inhibited by (S)-4-alkylthioamphetamine derivatives: insights from molecular modeling studies. Bioorg. Med. Chem. 15, 5198-5206. doi: 10.1016/j.bmc.2007.05.021

Finberg, J. P., and Rabey, J. M. (2016). Inhibitors of MAO-A and MAO-B in Psychiatry and Neurology. Front. Pharmacol. 7, 340. doi: 10.3389/ fphar.2016.00340

Fleckenstein, A. E., Volz, T. J., Riddle, E. L., Gibb, J. W., and Hanson, G. R. (2007). New insights into the mechanism of action of amphetamines. Annu. Rev. Pharmacol. Toxicol. 47, 681-698. doi: 10.1146/annurev.pharmtox. 47.120505.105140

Florvall, L., Fagervall, I., Ask, A. L., and Ross, S. B. (1986). Selective monoamine oxidase inhibitors. 4. 4-Aminophenethylamine derivatives with neuronselective action. J. Med. Chem. 29, 2250-2256. doi: 10.1021/jm00161a020

Fowler, C. J., and Oreland, L. (1981). Substrate- and stereoselective inhibition of human brain monoamine oxidase by 4 -dimethylamino-alpha, 2 dimethylphenethylamine (FLA 336). J. Pharm. Pharmacol. 33, 403-406. doi: 10.1111/j.2042-7158.1981.tb13819.x
Fresqui, M. A., Ferreira, M. M., and Trsic, M. (2013). The influence of R and S configurations of a series of amphetamine derivatives on quantitative structure-activity relationship models. Anal. Chim. Acta 759, 43-52. doi: 10.1016/j.aca.2012.11.004

Fuller, R. W., Baker, J. C., Perry, K. W., and Molloy, B. B. (1975). Comparison of 4chloro-, 4-bromo- and 4-fluoroamphetamine in rats: drug levels in brain and effects on brain serotonin metabolism. Neuropharmacology 14, 739-746. doi: 10.1016/0028-3908(75)90099-4

Gallardo-Godoy, A., Fierro, A., McLean, T. H., Castillo, M., Cassels, B. K., ReyesParada, M., et al. (2005). Sulfur-substituted alpha-alkyl phenethylamines as selective and reversible MAO-A inhibitors: biological activities, CoMFA analysis, and active site modeling. J. Med. Chem. 48, 2407-2419. doi: 10.1021/jm0493109

Glennon, R. A. (1999). Arylalkylamine drugs of abuse: an overview of drug discrimination studies. Pharmacol. Biochem. Behav. 64, 251-256. doi: 10.1016/ s0091-3057(99)00045-3

Gobbi, M., Funicello, M., Gerstbrein, K., Holy, M., Moya, P. R., Sotomayor, R., et al. (2008). N,N-dimethyl-thioamphetamine and methyl-thioamphetamine, two non-neurotoxic substrates of 5-HT transporters, have scant in vitro efficacy for the induction of transporter-mediated 5-HT release and currents. J. Neurochem. 105, 1770-1780. doi: 10.1111/j.1471-4159.2008.05272.x

Green, A. L., and el Hait, M. A. S. (1980). p-Methoxyamphetamine, a potent reversible inhibitor of type-A monoamine oxidase in vitro and in vivo. $J$. Pharm. Pharmacol. 32 262-266. doi: 10.1111/j.2042-7158.1980.tb12909.x.

Heal, D. J., Smith, S. L., Gosden, J., and Nutt, D. J. (2013). Amphetamine, past and present - a pharmacological and clinical perspective. J. Psychopharmacol. 27, 479-496. doi: 10.1177/0269881113482532

Hilbert, A. (2019). Binge-eating disorder. Psychiatr. Clin. North Am. 42, 33-43. doi: 10.1016/j.psc.2018.10.011

Hurtado-Guzmán, C., Fierro, A., Iturriaga-Vásquez, P., Sepúlveda-Boza, S., Cassels, B. K., and Reyes-Parada, M. (2003). Monoamine oxidase inhibitory properties of optical isomers and $\mathrm{N}$-substituted derivatives of 4 methylthioamphetamine. J. Enzyme Inhib. Med. Chem. 18, 339-347. doi: $10.1080 / 1475636031000118437$

Iacovino, L. G., Magnani, F., and Binda, C. (2018). The structure of monoamine oxidases: past, present, and future. J. Neural Transm (Vienna). 125, 1567-1579. doi: 10.1007/s00702-018-1915-z

Johnston, J. P. (1968). Some observations upon a new inhibitor of monoamine oxidase in brain tissue. Biochem. Pharmacol. 17, 1285-1297. doi: 10.1016/00062952(68)90066-x

Kalgutkar, A. S., Dalvie, D. K., Castagnoli., N.Jr., and Taylor, T. J. (2001). Interactions of nitrogen-containing xenobiotics with monoamine oxidase (MAO) isozymes A and B: SAR studies on MAO substrates and inhibitors. Chem. Res. Toxicol. 14, 1139-1162. doi: 10.1021/tx010073b

Kilpatrick, I. C., Traut, M., and Heal, D. J. (2001). Monoamine oxidase inhibition is unlikely to be relevant to the risks associated with phentermine and fenfluramine: a comparison with their abilities to evoke monoamine release. Int. J. Obes. Relat. Metab. Disord. 25, 1454-1458. doi: 10.1038/sj.ijo.0801732

Kinemuchi, H., Arai, Y., Toyoshima, Y., Tadano, T., and Kisara, K. (1988). Studies on 5-fluoro-alpha-methyltryptamine and p-chloro-betamethylphenethylamine: determination of the MAO-A or MAO-B selective inhibition in vitro. Jpn. J. Pharmacol. 46 197-199. doi: 10.1254/jjp.46.197

Kumar, B., Gupta, V. P., and Kumar, V. A. (2017). Perspective on monoamine oxidase enzyme as drug target: challenges and opportunities. Curr. Drug Targets. 18, 87-97. doi: 10.2174/1389450117666151209123402

Lamberth, P. G., Ding, G. K., and Nurmi, L. A. (2008). Fatal paramethoxyamphetamine (PMA) poisoning in the Australian Capital Territory. Med. J. Aust. 188, 426. doi: 10.5694/j.1326-5377.2008.tb01695.x

Lapoint, J., Dargan, P. I., and Hoffman, R. S. (2013). "Synthetic Amphetamine Derivatives," in Novel Psychoactive Substances. Eds. P. I. Dargan and D. M. Wood (Boston, MA: Academic Press), 161-178.

Leonardi, E. T., and Azmitia, E. C. (1994). MDMA (ecstasy) inhibition of MAO type A and type B: comparisons with fenfluramine and fluoxetine (Prozac). Neuropsychopharmacology 10, 231-238. doi: 10.1038/npp.1994.26

Luethi, D., and Liechti, M. E. (2018). Monoamine transporter and receptor interaction profiles in vitro predict reported human doses of novel psychoactive stimulants and psychedelics. Int. J. Neuropsychopharmacol. 21, 926-931. doi: 10.1093/ijnp/pyy047 
Luque, J. M., Kwan, S. W., Abell, C. W., Da Prada, M., and Richards, J. G. (1995). Cellular expression of mRNAs encoding monoamine oxidases A and B in the rat central nervous system. J. Comp. Neurol. 363, 665-680. doi: 10.1002/ cne.903630410

Ma, J., Masato, Y., Yamashita, E., Nakagawa, A., Ito, A., and Tsukihara, T. (2004). Structure of rat monoamine oxidase and its specific recognitions for substrates and inhibitors. J. Mol. Biol. 338, 103-114. doi: 10.1016/j.jmb.2004.02.032

Magyar, K. (1994). Behaviour of (-)-deprenyl and its analogues. J. Neural Transm. Suppl. 41, 167-175. doi: 10.1007/978-3-7091-9324-2_23

Malcomson, T., Yelekci, K., Borrello, M. T., Ganesan, A., Semina, E., De Kimpe, N., et al. (2015). cis-Cyclopropylamines as mechanism-based inhibitors of monoamine oxidases. FEBS J. 282, 3190-3198. doi: 10.1111/febs.13260

Mantle, T. J., Tipton, K. F., and Garrett, N. J. (1976). Inhibition of monoamine oxidase by amphetamine and related compounds. Biochem. Pharmacol. 25, 2073-2077. doi: 10.1016/0006-2952(76)90432-9

Markou, A., Kosten, T. R., and Koob, G. F. (1998). Neurobiological similarities in depression and drug dependence: a self-medication hypothesis. Neuropsychopharmacology 18, 135-174. doi: 10.1016/S0893-133X(97)00113-9

Martin, T. L. (2001). Three cases of fatal paramethoxyamphetamine overdose. J. Anal. Toxicol. 25, 649-651. doi: 10.1093/jat/25.7.649

Matsumoto, T., Maeno, Y., Kato, H., Seko-Nakamura, Y., Monma-Ohtaki, J., Ishiba, A., et al. (2014). 5-Hydroxytryptamine- and dopamine-releasing effects of ring-substituted amphetamines on rat brain: a comparative study using in vivo microdialysis. Eur. Neuropsychopharmacol. 24, 1362-1370. doi: 10.1016/ j.euroneuro.2014.04.009

Miller, J. R., and Edmondson, D. E. (1999). Structure-activity relationships in the oxidation of para-substituted benzylamine analogues by recombinant human liver monoamine oxidase A. Biochemistry38, 13670-13683. doi: 10.1021/ bi990920y

Núñez-Vivanco, G., Fierro, A., Moya, P., Iturriaga-Vásquez, P., and Reyes-Parada, M. (2018). 3D similarities between the binding sites of monoaminergic target proteins. PloS One 13, e0200637. doi: 10.1371/journal.pone.0200637

Nandigama, R. K., Newton-Vinson, P., and Edmondson, D. E. (2002). Phentermine inhibition of recombinant human liver monoamine oxidases A, and B. Biochem. Pharmacol. 63, 865-869. doi: 10.1016/s0006-2952(02)00840-7

Nichols, D. E., Marona-Lewicka, D., Huang, X., and Johnson, M. P. (1993). Novel serotonergic agents. Drug Des. Discov. 9, 299-312.

Nichols, D. E. (1994). "Medicinal Chemistry and Structure-Activity Relationships," in Amphetamine and Its Analogues: Psychopharmacology, Toxicology, and Abuse. Eds. A. K. Cho and D. S. Segal (San Diego, CA: Academic Press), 3-41.

Nichols, D. E. (2018). Chemistry and Structure-Activity Relationships of Psychedelics. Curr. Top. Behav. Neurosci. 36, 1-43. doi: 10.1007/7854_2017_475

Norinder, U., Florvall, L., and Ross, S.B. (1994). A PLS quantitative structureactivity relationship study of some monoamine oxidase inhibitors of the phenyl alkylamine type. Eur. J. Med. Chem. 29, 191-195. doi: 10.1016/0223-5234(94) 90037-X

Osorio-Olivares, M., Rezende, M. C., Sepúlveda-Boza, S., Cassels, B. K., and Fierro, A. (2004). MAO inhibition by arylisopropylamines: the effect of oxygen substituents at the beta-position. Bioorg. Med. Chem. 12, 4055-4066. doi: 10.1016/ j.bmc.2004.05.033

Paillet-Loilier, M., Cesbron, A., Le Boisselier, R., Bourgine, J., and Debruyne, D. (2014). Emerging drugs of abuse: current perspectives on substituted cathinones. Subst. Abuse Rehabil. 5, 37-52. doi: 10.2147/SAR.S37257

Prinzmetal, M., and Bloomberg, W. (1935). The use of benzedrine for the treatment of narcolepsy. J. Am. Med. Assoc. 105, 2051-2054. doi: 10.1001/ jama.1935.02760510023006

Reyes-Parada, M., Scorza, M. C., Silveira, R., Dajas, F., Costa, G., Tipton, K. F., et al. (1994a). Monoamine oxidase inhibitory effects of some 4aminophenethylamine derivatives. Biochem. Pharmacol. 47, 1365-1371. doi: 10.1016/0006-2952(94)90335-2

Reyes-Parada, M., Scorza, M. C., Silveira, R., Dajas, F., and Cassels, B. K. (1994b). 4-Dimethylaminophenethylamine, a sensitive, specific, electrochemically detectable monoamine oxidase-B substrate. Life Sci. 54, 1955-1963. doi: 10.1016/0024-3205(94)90130-9

Reyes-Parada, M., Fierro, A., Iturriaga-Vásquez, P., and Cassels, B. K. (2005). Monoamine oxidase inhibition in the light of new structural data. Curr. Enz. Inhib. 1, 85-95. doi: 10.2174/1573408052952711
Robinson, J. B. (1985). Stereoselectivity and isoenzyme selectivity of monoamine oxidase inhibitors. Enantiomers of amphetamine, $\mathrm{N}$-methylamphetamine and deprenyl. Biochem. Pharmacol. 34, 4105-4108. doi: 10.1016/0006-2952(85) 90201-1

Rothman, R. B., and Baumann, M. H. (2003). Monoamine transporters and psychostimulant drugs. Eur. J. Pharmacol. 479, 23-40. doi: 10.1016/ j.ejphar.2003.08.054

Rothman, R. B., Blough, B. E., Woolverton, W. L., Anderson, K. G., Negus, S. S., Mello, N. K., et al. (2005). Development of a rationally designed, low abuse potential, biogenic amine releaser that suppresses cocaine self-administration. J. Pharmacol. Exp. Ther. 313, 1361-1369. doi: 10.1124/jpet.104.082503

Rothman, R. B., Blough, B. E., and Baumann, M. H. (2007). Dual dopamine/ serotonin releasers as potential medications for stimulant and alcohol addictions. AAPS J. 9, E1-E10. doi: 10.1208/aapsj0901001

Rothman, R. B., Blough, B. E., and Baumann, M. H. (2008). Dual dopamine/ serotonin releasers: potential treatment agents for stimulant addiction. Exp. Clin. Psychopharmacol. 16, 458-474. doi: 10.1037/a0014103

Santillo, M. F. (2014). Inhibition of monoamine oxidase (MAO) by $\alpha$ ethylphenethylamine and $\mathrm{N}, \alpha$-diethylphenethylamine, two compounds related to dietary supplements. Food Chem. Toxicol. 74, 265-269. doi: 10.1016/j.fct.2014.10.009

Scorza, M. C., Carrau, C., Silveira, R., Zapata-Torres, G., Cassels, B. K., and ReyesParada, M. (1997). Monoamine oxidase inhibitory properties of some methoxylated and alkylthio amphetamine derivatives: structure-activity relationships. Biochem. Pharmacol. 54, 1361-1369. doi: 10.1016/s0006-2952 (97)00405-x

Scorza, C., Silveira, R., Nichols, D. E., and Reyes-Parada, M. (1999). Effects of 5-HTreleasing agents on the extracellullar hippocampal 5-HT of rats. Implications for the development of novel antidepressants with a short onset of action. Neuropharmacology38, 1055-1061. doi: 10.1016/s0028-3908(99)00023-4

Shih, J. C., Chen, K., and Ridd, M. (1999). Monoamine oxidases: from genes to behavior. Ann. Rev. Neurosci. 22, 197-217. doi: 10.1146/annurev.neuro. 22.1.197

Shulgin, A. T., and Shulgin, A. (1991). Phenethylamines I Have Known And Loved (Berkeley: Transform Press).

Simmler, L., Buser, T., Donzelli, M., Schramm, Y., Dieu, L. H., Huwyler, J., et al. (2013). Pharmacological characterization of designer cathinones in vitro. Br. J. Pharmacol. 168458-470. doi: 10.1111/j.1476-5381.2012.02145.x

Sitte, H. H., and Freissmuth, M. (2015). Amphetamines, new psychoactive drugs and the monoamine transporter cycle. Trends Pharmacol. Sci. 36, 41-50. doi: 10.1016/j.tips.2014.11.006

Son, S. Y., Ma, J., Kondou, Y., Yoshimura, M., Yamashita, E., and Tsukihara, T. (2008). Structure of human monoamine oxidase A at 2.2- $\AA$ resolution: the control of opening the entry for substrates/inhibitors. Proc. Natl. Acad. Sci. U. S. A. 105, 5739-5744. doi: 10.1073/pnas.0710626105

Sotomayor-Zárate, R., Quiroz, G., Araya, K. A., Abarca, J., Ibáñez, M. R., Montecinos, A., et al. (2012). 4-Methylthioamphetamine increases dopamine in the rat striatum and has rewarding effects in vivo. Basic Clin. Pharmacol. Toxicol. 111371-379. doi: 10.1111/j.1742-7843.2012.00926.x

Steuer, A. E., Boxler, M. I., Stock, L., and Kraemer, T. (2016). Inhibition potential of 3,4-methylenedioxymethamphetamine (MDMA) and its metabolites on the in vitro monoamine oxidase (MAO)-catalyzed deamination of the neurotransmitters serotonin and dopamine. Toxicol. Lett. 243, 48-55. doi: 10.1016/j.toxlet.2015.12.001

Sulzer, D., Sonders, M. S., Poulsen, N. W., and Galli, A. (2005). Mechanisms of neurotransmitter release by amphetamines: a review. Prog. Neurobiol. 75, 406-433. doi: 10.1016/j.pneurobio.2005.04.003

Tipton, K. F., Boyce, S., O'Sullivan, J., Davey, G. P., and Healy, J. (2004). Monoamine oxidases: certainties and uncertainties. Curr. Med. Chem. 11, 1965-1982. doi: 10.2174/0929867043364810

Tripathi, A. C., Upadhyay, S., Paliwal, S., and Saraf, S. K. (2018). Privileged scaffolds as MAO inhibitors: Retrospect and prospects. Eur. J. Med. Chem. 145, 445-497. doi: 10.1016/j.ejmech.2018.01.003

Ulus, I. H., Maher, T. J., and Wurtman, R. J. (2000). Characterization of phentermine and related compounds as monoamine oxidase (MAO) inhibitors. Biochem. Pharmacol. 59, 1611-1621. doi: 10.1016/s0006-2952(00)00306-3

Vallejos, G., Rezende, M. C., and Cassels, B. K. (2002). Charge-transfer interactions in the inhibition of MAO-A by phenylisopropylamines - a 
QSAR study. J. Comput. Aided Mol. Des. 16, 95-103. doi: 10.1023/ a:1016344030772

Vallejos, G., Fierro, A., Rezende, M. C., Sepúlveda-Boza, S., and Reyes-Parada, M. (2005). Heteroarylisopropylamines as MAO inhibitors. Bioorg. Med. Chem. 13, 4450-4457. doi: 10.1016/j.bmc.2005.04.045

Vianello, R., Domene, C., and Mavri, J. (2016). The use of multiscale molecular simulations in understanding a relationship between the structure and function of biological systems of the brain: the application to monoamine oxidase enzymes. Front. Neurosci. 10, 327. doi: 10.3389/fnins.2016.00327

Vilches-Herrera, M., Miranda-Sepúlveda, J., Rebolledo-Fuentes, M., Fierro, A., Lühr, S., Iturriaga-Vasquez, P., et al. (2009). Naphthylisopropylamine and Nbenzylamphetamine derivatives as monoamine oxidase inhibitors. Bioorg. Med. Chem. 17, 2452-2460. doi: 10.1016/j.bmc.2009.01.074

Vilches-Herrera, M., Fierro, A., Lühr, S., Miranda-Sepúlveda, J., Iturriaga-Vasquez, P., Reyes-Parada, M., et al. (2016). Cycloalkylthiophenylisopropylamine derivatives are better monoamine oxidase inhibitors than their open chain analogues. Rev. Farmacol. Chile. 9, 39-42.

Wagmann, L., Brandt, S. D., Kavanagh, P. V., Maurer, H. H., and Meyer, M. R. (2017). In vitro monoamine oxidase inhibition potential of alphamethyltryptamine analog new psychoactive substances for assessing possible toxic risks. Toxicol. Lett. 272, 84-93. doi: 10.1016/j.toxlet.2017.03.007

Welter-Luedeke, J., and Maurer, H. H. (2016). New Psychoactive Substances: Chemistry, Pharmacology, Metabolism, and Detectability of Amphetamine Derivatives With Modified Ring Systems. Ther. Drug Monit. 38, 4-11. doi: 10.1097/FTD.0000000000000240

Westlund, K. N., Denney, R. M., Rose, R. M., and Abell, C. W. (1988). Localization of distinct monoamine oxidase $\mathrm{A}$ and monoamine oxidase $\mathrm{B}$ cell populations in human brainstem. Neuroscience25, 439-456. doi: 10.1016/0306-4522(88)90250-3

Wouters, J. (1998). Structural aspects of monoamine oxidase and its reversible inhibition. Curr. Med. Chem. 5, 137-162.

Yoshida, S., Rosen, T. C., Meyer, O. G., Sloan, M. J., Ye, S., Haufe, G., et al. (2004). Fluorinated phenylcyclopropylamines. Part 3: Inhibition of monoamine oxidase A, and B. Bioorg. Med. Chem. 12, 2645-2652. doi: 10.1016/ j.bmc.2004.03.010
Youdim, M. B. H., Edmondson, D., and Tipton, K. F. (2006). The therapeutic potential of monoamine oxidase inhibitors. Nat. Rev. Neurosci. 7, 295-309. doi: $10.1038 / \mathrm{nrn} 1883$

Yu, P. H., Bailey, B. A., Durden, D. A., and Boulton, A. A. (1986). Stereospecific deuterium substitution at the alpha-carbon position of dopamine and its effect on oxidative deamination catalyzed by MAO-A and MAO-B from different tissues. Biochem. Pharmacol. 35, 1027-1036. doi: 10.1016/0006-2952(86) 90094-8

Yu, P. H., Davis, B. A., Boulton, A. A., and Zuo, D. M. (1994). Deamination of aliphatic amines by type B monoamine oxidase and semicarbazide-sensitive amine oxidase; pharmacological implications. J. Neural Transm. Suppl. 41, 397-406. doi: 10.1007/978-3-7091-9324-2_53

Yu, P. H., Davis, B. A., and Boulton, A. A. (1995). Aliphatic propargylamines, a new series of potent selective, irreversible non-amphetamine-like MAO-B inhibitors. Their structures, function and pharmacological implications. Adv. Exp. Med. Biol. 363, 17-23. doi: 10.1007/978-1-4615-1857-0_3

$\mathrm{Yu}$, P. H. (1986). Inhibition of monoamine oxidase activity by phenylpropanolamine, an anorectic agent. Res. Commun. Chem. Pathol. Pharmacol. 51, 163-171.

Yu, P. H. (1988). Three types of stereospecificity and the kinetic deuterium isotope effect in the oxidative deamination of dopamine as catalyzed by different amine oxidases. Biochem. Cell Biol. 66, 853-861. doi: 10.1139/o88-097

Conflict of Interest: The authors declare that the research was conducted in the absence of any commercial or financial relationships that could be construed as a potential conflict of interest.

Copyright (c) 2020 Reyes-Parada, Iturriaga-Vasquez and Cassels. This is an openaccess article distributed under the terms of the Creative Commons Attribution License (CC BY). The use, distribution or reproduction in other forums is permitted, provided the original author(s) and the copyright owner(s) are credited and that the original publication in this journal is cited, in accordance with accepted academic practice. No use, distribution or reproduction is permitted which does not comply with these terms. 